\title{
OPEN Circulating acyl and des-acyl ghrelin levels in obese adults: a systematic review and meta-analysis
}

\author{
Yanmei Wang ${ }^{1,2,4}$, Qianxian $\mathrm{Wu}^{2,4}$, Qian $\mathrm{Zhou}^{1}$, Yuyu Chen ${ }^{3}$, Xingxing Lei ${ }^{1}$, Yiding Chen ${ }^{1}$ \& \\ Qiu Chen ${ }^{1 \bowtie}$
}

Ghrelin is the only known orexigenic gut hormone, and its synthesis, secretion and degradation are affected by different metabolic statuses. This meta-analysis aimed to investigate the potential differences in plasma acyl ghrelin (AG) and des-acyl ghrelin (DAG) concentrations between normal weight and obese adults. Systematic literature searches of PubMed, Embase and Web of Science through October 2021 were conducted for articles reporting AG or DAG levels in obesity and normal weight, and 34 studies with 1863 participants who met the eligibility criteria were identified. Standardized mean differences (SMDs) with $95 \%$ confidence intervals (Cls) were calculated to evaluate group differences in circulating AG and DAG levels. Pooled effect size showed significantly lower levels of baseline AG (SMD: $-0.85 ; 95 \% \mathrm{Cl}:-1.13$ to $-0.57 ; P_{\text {SMD }}<0.001$ ) and DAG (SMD: $-1.06 ; 95 \%$ $\mathrm{Cl}:-1.43$ to $\left.-0.69 ; P_{\mathrm{SMD}}<0.001\right)$ in obese groups compared with healthy controls, and similar results were observed when subgroup analyses were stratified by the assay technique or storage procedure. Postprandial AG levels in obese subjects were significantly lower than those in controls when stratified by different time points $\left(\mathrm{SMD}_{30 \mathrm{~min}}:-0.85,95 \% \mathrm{Cl}:-1.18\right.$ to $-0.53, P_{\mathrm{SMD}}<0.001 ; \mathrm{SMD}_{60 \mathrm{~min}}:-1.00$, $95 \% \mathrm{Cl}:-1.37$ to $-0.63, P_{\mathrm{SMD}}<0.001$; SMD $120 \mathrm{~min}$ : $-1.21,95 \% \mathrm{Cl}:-1.59$ to $\left.-0.83, P_{\mathrm{SMD}}<0.001\right)$. In healthy subjects, a postprandial decline in AG was observed at $120 \mathrm{~min}$ (SMD: $-0.42 ; 95 \% \mathrm{Cl}:-0.77$ to $-0.06 ; P_{S M D}=0.021$ ) but not in obese subjects (SMD: $-0.28 ; 95 \% \mathrm{Cl}:-0.60$ to $0.03 ; P_{S M D}=0.074$ ). The mean change in AG concentration was similar in both the obese and lean health groups at each time point $\left(\Delta \mathrm{SMD}_{30 \mathrm{~min}}: 0.31,95 \% \mathrm{Cl}:-0.35\right.$ to $0.97, P_{\mathrm{SMD}}=0.359 ; \Delta \mathrm{SMD}_{60 \mathrm{~min}}: 0.17,95 \% \mathrm{Cl}:-0.12$ to 0.46 , $P_{S M D}=0.246 ; \Delta S S_{120 \min }: 0.21,95 \% \mathrm{Cl}:-0.13$ to $\left.0.54, P_{S M D}=0.224\right)$. This meta-analysis strengthens the clinical evidence supporting the following: lower baseline levels of circulating AG and DAG in obese individuals; declines in postprandial circulating AG levels, both for the healthy and obese individuals; a shorter duration of AG suppression in obese subjects after meal intake. These conclusions have significance for follow-up studies to elucidate the role of various ghrelin forms in energy homeostasis.

Obesity is defined by the Centers for Disease Control and Prevention (CDC) ${ }^{1}$ as a body mass index $\geq 30 \mathrm{~kg} /$ $\mathrm{m}^{2}$, and has become a global epidemic with the improvement of living standards. As the major risk factor for a large number of serious complications, individuals with obesity are more likely to have diabetes mellitus, dyslipidemia, hypertension, nonalcoholic fatty liver, cardiovascular disease, cancer and severe coronavirus disease 2019 (COVID-19), which lead to a higher rate of adult mortality ${ }^{2,3}$. Obesity is a multifactorial disease that is particularly associated with malfunctioning signal mechanisms. Complex signaling systems regulate energy homeostasis, where gastrointestinal hormones have a central physiological function.

Ghrelin is a gut hormone with the strongest orexigenic signal ${ }^{4}$ that helps the body respond to changes in metabolic status by binding to growth hormone secretagogue receptors (GHSRs) expressed in multiple central and peripheral targets ${ }^{5,6}$, with actions that include an increase in caloric intake, downregulation of energy

\footnotetext{
${ }^{1}$ Hospital of Chengdu University of Traditional Chinese Medicine, No. 39 Shi-er-qiao Road, Jinniu District, Chengdu 610075, Sichuan, China. ${ }^{2}$ Ya'an Polytechnic College, No. 130 Yucai Road, Yucheng District, Yaan 625000, Sichuan, China. ${ }^{3} \mathrm{Halifa}$ Regional Centre for Education, No. 33 Spectacle Lake Dr, Dartmouth, NS B3B1X7, Canada. ${ }^{4}$ These authors contributed equally: Yanmei Wang and Qianxian Wu. ${ }^{\varpi}$ email: chenqiu1005@ cdutcm.edu.cn
} 
expenditure $^{7}$, potentiation of growth hormone $(\mathrm{GH})$ release $^{8}$, stimulation of gastric emptying and motility ${ }^{6,9}$, and anti-depressant-like properties ${ }^{6}$.

Endogenous ghrelin in adults is produced predominantly by P/D1 cells, which are located in the oxyntic glands of gastric funds ${ }^{10,11}$. Following secretion into the bloodstream, ghrelin circulates in two major forms: acyl ghrelin (AG) and des-acyl ghrelin (DAG, also known as unacyl ghrelin), and the ratio of the former to the latter is approximately $10 \%$ in plasma ${ }^{12,13}$. AG is a 28 amino acid peptide hormone often seen as the active form of ghrelin because of its unique posttranslational acylation at the serine 3 residue, which is catalyzed by ghrelin$\mathrm{O}$-acyltransferase (GOAT) and is essential for binding a GHSR with high affinity ${ }^{14,15}$. Without acylation, ghrelin can be secreted directly in the form of DAG. Furthermore, DAG is considered to be a degradation product of acyl ghrelin in the circulation due to esterase-catalyzed deacylation by multiple plasma proteins, especially acyl-protein thioesterase 1 (APT1) ${ }^{16}$ and butyrylcholinesterase (BChE) ${ }^{17,18}$. Although des-acyl ghrelin does not activate GHSR at physiological ranges ${ }^{11}$, emerging evidence has shown its independent biological activity, which may antagonize the orexigenic effects of acyl ghrelin in some instances ${ }^{19-22}$ even if the receptors and mechanisms remain undefined ${ }^{20,23-26}$.

The synthesis, secretion and degradation of ghrelin are affected by different metabolic statuses ${ }^{27}$. Current knowledge regarding circulating ghrelin levels describes that circulating ghrelin levels elevated during shortterm fasting and decreased upon meal ingestion in healthy humans ${ }^{6,28,29}$, which is consistent with its unique mechanism of action on orexigenic hormone evolution for energy storage and seeking ${ }^{30}$. Thus, ghrelin is believed to increase the risk of obesity. Contrary to expectations, people with obesity usually exhibit lower fasting levels of ghrelin ${ }^{31,32}$ with a decline in postprandial suppression ${ }^{33}$, and different published studies that focus on obesity have reported a negative correlation between plasma ghrelin levels and body mass index ${ }^{31,32}$. This abnormality may result from physiological adaptation with a positive energy balance in obese participants ${ }^{31}$; however, it is important to note that merely evaluating total ghrelin could not reflect the real metabolic status in obesity, since acyl and des-acyl ghrelin interact with different receptors and appear to have opposite actions. The decrease in ghrelin may be attributed to the balance change between the two forms or significant DAG reduction. Given the rapid deacylation of AG to DAG by plasma esterases and the limitations of assay methods, the accurate assessment of circulating ghrelin has proven to be challenging, and only a few studies have assessed both forms independently. Following the commercialization of sandwich ELISA kits and the standardization of collection, handling and storage of biological samples, it is possible to distinguish and measure the two different forms correctly $^{34,35}$. However, recent observations have shown inconsistent results in the circulating levels of AG and DAG in individuals with obesity, reflected not only at baseline but also at postprandial levels ${ }^{36-44}$.

Soon after its discovery, "the hunger hormone" 45 attracted increasing interest in the treatment of obesity and related diseases. To date, GHSR antagonists, ghrelin vaccines and GOAT inhibitors have shown some promise for weight loss, calorie reduction and energy expenditure. Nonetheless, the maintenance of energy homeostasis through the ghrelin system is far more complicated than previously appreciated, and simply suppressing or reducing ghrelin has not achieved the desired treatment goal in human trials $s^{46-49}$.

Thus, a full understanding of the difference in the biochemical composition of plasma ghrelin under different dietetic states between normal weight and obese individuals is indispensable before the identification of pharmacological targets in ghrelin signaling. Therefore, the aim of this study was to conduct a meta-analysis of all eligible published articles to independently investigate the potential differences in plasma AG and DAG concentrations between normal weight and obese adults.

\section{Methods}

The report and conduct of this systematic review and meta-analysis was based on the PRISMA (Preferred Reporting Items for Systematic Reviews and Meta-Analysis) statement, and a protocol has been registered in PROSPERO (International Prospective Register of Systematic Reviews) with the registration number CRD42021247253.

Literature search. Literature searches were conducted based on three online electronic bibliographic databases, PubMed, EMBASE and Web of Science, from their date of inception up to 22 October 2021. We used Medical Subject Headings (MeSH) words of "obesity" and the free terms to represent the disease, the key words "acylghrelin" OR "acyl ghrelin" OR "acyl-ghrelin" OR "active ghrelin" OR "active-ghrelin" OR "acylated ghrelin” OR “acylated-ghrelin” OR "desacylghrelin” OR "desacyl ghrelin” OR “desacyl-ghrelin” OR "des-acyl ghrelin” OR “des-n-octanoyl ghrelin” OR “unacyl ghrelin” OR "unacyl-ghrelin” OR "unacylated ghrelin” OR "unacylated-ghrelin" OR "des-acylated ghrelin" OR "desacylated-ghrelin" OR "desacylated ghrelin" OR "nonaclyated ghrelin" OR "nonacylated ghrelin" OR "nonacylated-ghrelin" as our target. To ensure maximum eligible study coverage, the reference lists of pertinent articles were inspected manually. The full search strategies for all databases can be found in Supplementary Table 1. Two authors (YM Wang and QX Wu) independently screened and cross-checked the literature by identifying all titles and abstracts. Then, the selected articles were reviewed in full to ensure compliance with the inclusion criteria. A third author (Q Chen) was consulted regarding the disagreements. Specific libraries were created to allow the identification and exclusion of duplicate studies and the division and organization of the results.

Selection criteria. (1) Articles studying the circulating acyl or des-acyl ghrelin levels in obese humans aged 18 to 80 years; (2) BMI was used to define obesity with the following standards ${ }^{1}$ : normal weight: 18.5 to $<25 \mathrm{~kg} / \mathrm{m}^{2}$, overweight: 25.0 to $<30 \mathrm{~kg} / \mathrm{m}^{2}$, obesity: $\geq 30 \mathrm{~kg} / \mathrm{m}^{2}$. Both overweight and obesity were allocated to case group; (3) Acyl or des-acyl ghrelin levels were measured after an overnight fasting (with or without postprandial concentrations); (4) Specific weight loss interventions on obesity, such as drugs, surgeries, regular exercises were disallowed before the measurement; (5) People included in these studies were in a relatively 
healthy condition, without genetic disorders known to cause obesity, eating disorders, heart disease, cancer, severe hepatic or renal disease, pregnancy, confirmed diagnosis of diabetes mellitus, uncontrolled hypertension, et al. To ensure maximum coverage of eligible studies, obese patients with metabolic syndrome (MetS, which was defined as the presence of three or more of following diagnostic criteria: abdominal obesity and waist circumference $\geq 88 \mathrm{~cm}$ for women or $\geq 102 \mathrm{~cm}$ for men; fasting plasma glucose $>6.1 \mathrm{mmol} / \mathrm{L}$; circulating triglycerides $\geq 1.7 \mathrm{mmol} / \mathrm{L}$; high-density lipoprotein $<40 \mathrm{mg} / \mathrm{dl}$ in men or $<50 \mathrm{mg} / \mathrm{dl}$ in women; hypertension including systolic pressure $\geq 140 \mathrm{mmHg}$ or diastolic pressure $\geq 90 \mathrm{mmHg}$ or antihypertensive treatment, according to the criteria of the National Cholesterol Education Program Adult Treatment Panel III guidelines ${ }^{50,51}$ were included; (6) More than 6 points of Newcastle-Ottawa Scale (NOS) ${ }^{52}$ score were considered eligible for inclusion.

Exclusion criteria. (1) Studies that only measured total ghrelin and failed to assess acyl and des-acyl ghrelin levels independently; (2) Abstracts, case reports, reviews or nonclinical studies; (3) Studies that were not written in English; (4) Studies lacking a healthy weight control group; (5) Studies that had duplicate data or repeat analysis; (6) The sample size of original articles was less than 10; (7) The data not presented as or could not be converted to the form of mean \pm standard deviation $(\mathrm{SD})$.

Quality assessment and data collection. Quality assessment of the included articles was performed according to The Newcastle-Ottawa Quality Assessment Scale (NOS), which was designed to target nonrandomized studies and contains three different types of biases: bias of selection (0-4), bias of comparability (0-2) and bias of exposure (0-3). Studies with more than 6 points on the NOS score were considered eligible for inclusion $^{52}$. A pretested standardized form was used to extract data from the included studies for study evaluation and evidence synthesis. The descriptive details included authors, population, sample size, sex, sample age, blood sample, handling methods, measuring methods, types of test meals, fasting and postprandial ghrelin levels. Both quality assessment and data extraction were also conducted independently by two reviewer authors (YM Wang and QX Wu), and discrepancies were identified and resolved through discussion with a third author (Q Chen).

Statistical considerations. Stata/SE 15.0 for Mac (Stata Corp, College Station, TX, USA) was used to analyze the statistical data. The fasting and postprandial AG or DAG levels were summarized for each study sample and reported as the mean and the standard deviation (SD). Data presented as standard error (SE) were converted to $\mathrm{SD}$ by the equation $\mathrm{SD}=\mathrm{SE} \times \sqrt{\text { number of subjects; }}$ moreover, when median and interquartile range appeared, a validated procedure was adopted to convert ${ }^{53}$ before being entered. Plasma DAG was calculated by subtracting AG from total ghrelin (TG) ${ }^{54,55}$ when studies happened to report AG and TG alone. As needed, data were obtained from graphs using Engauge Digitizer 12.1. The postprandial time points were chosen for consistency across the study protocols to allow for comparison. The changes in hormone concentrations from baseline to postprandial states were calculated as follows ${ }^{56}$ : mean difference $=$ mean at postprandial - mean at baseline, standard deviation of mean difference $=\sqrt{\mathrm{SD}_{\text {baseline }}^{2}+\mathrm{SD}_{\text {postprandial }}^{2}-2 \times \mathrm{r} \times \mathrm{SD}_{\text {baseline }} \times \mathrm{SD}_{\text {postprandial }}}$, considering a correlation coefficient $(\mathrm{r})$ of 0.5. When multiple relevant groups existed, formulas in the Cochrane Handbook were used to calculate the combined mean and $\mathrm{SD}^{57}$. Due to the different measuring methods with various units for ghrelin, continuous variables were expressed as standardized mean differences (SMDs) with 95\% confidence intervals (CIs). $P_{\mathrm{SMD}}<0.05$ for any test or model was considered statistically significant. The $\mathrm{I}^{2}$ statistic and Cochrane's Q test were measured to analyze the heterogeneity, and the cutoff values were $50 \%$ and 0.05 , respectively. A fixed-effect model was used for the meta-analysis with moderate heterogeneity $\left(\mathrm{I}^{2}<50 \%, P_{\text {heterogeneity }}>0.05\right)$; otherwise, a random-effects model was performed, and a Galbraith plot was used to detect potential sources of heterogeneity. Subgroup analyses were performed according to blood sample handling and measuring methods. Publication bias was inspected by Begg's funnel plots and Egger's linear regression test when more than ten studies were involved, and a $P$ value $<0.05$ indicated potential publication bias.

\section{Results}

Study selection. The PRISMA statement flow diagram outlines the procedures of literature identification, screening and study exclusion (Fig. 1). A total of 5209 putative articles were initially retrieved. After the removal of duplicates, reviewing titles and abstracts, and reading through full texts, 34 eligible articles ${ }^{37-44,58-83}$ that met the selection criteria were included in our systematic review and meta-analysis. The quality assessment of these studies is presented in Supplementary Fig. 1. All of the included studies had an NOS score over 6 points, which was considered high-quality.

General characteristics. The general characteristics of the included studies are described in Table 1. An aggregate of 1863 adult participants (1125 obese patients and 738 healthy controls) were investigated in the 34 included trials. The mean participant BMI of each study ranged from 27.4 to $49.4 \mathrm{~kg} / \mathrm{m}^{2}$ for patients and 18.5 to $23.2 \mathrm{~kg} / \mathrm{m}^{2}$ for healthy controls. One Singapore ${ }^{44}$ study defined obesity with a BMI above $27.5 \mathrm{~kg} / \mathrm{m}^{2}$ and healthy lean subjects with a BMI ranging from 18.5 to $23 \mathrm{~kg} / \mathrm{m}^{2}$. For the remaining articles, BMI definition was based on the CDC criteria, and 6 articles s $^{38,61,65,76,78,79}$ included both obese patients and overweight subjects. All included participants had no genetic diseases identified as the cause of their obesity, and diabetes mellitus was considered an exclusion criterion, but obesity with MetS ${ }^{40,64,74,80,83}$ or obesity with morbidity ${ }^{64,79,81}$ and hypertension or hyperlipidemia under control by drugs ${ }^{69,82}$ were included. 


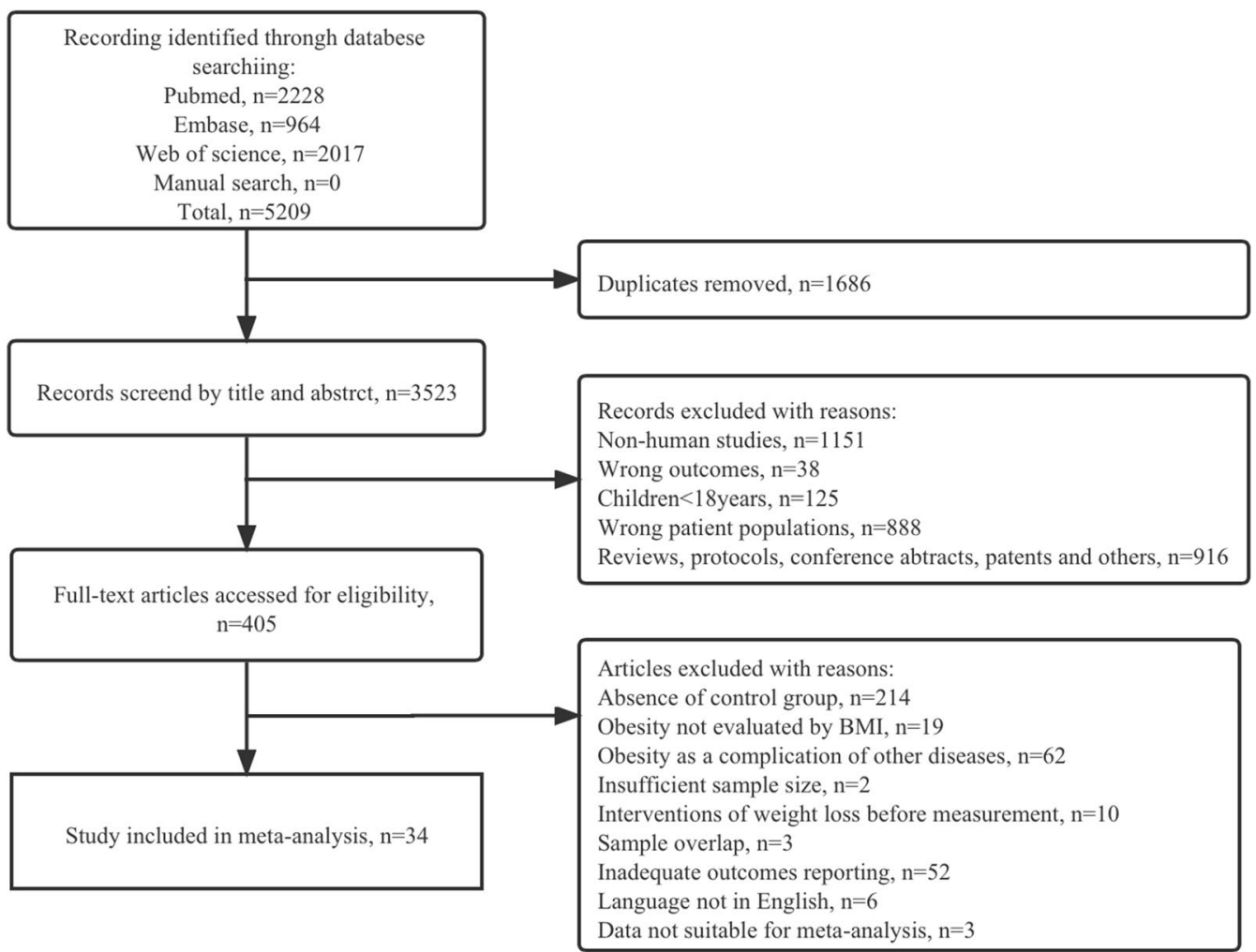

Figure 1. Flow diagram of the literature search and study selection process.

Fasting AG. Thirty-three included studies ${ }^{37-44,58-79,81-83}$ with 1066 obese cases and 717 healthy controls measured baseline fasting circulating acyl ghrelin levels in obese patients and control subjects. The pooled effect size showed significantly lower levels of baseline AG in the obese groups than in the healthy controls (SMD:-0.85; 95\% CI: -1.13 to $-0.57 ; P_{\text {SMD }}<0.001$ ) (Fig. 2 and Table 2). Interstudy heterogeneity was significant, with an $\mathrm{I}^{2}$ of $86.4 \%\left(P_{\text {heterogeneity }}<0.001\right)$, and a random-effects model was applied. Ten studies ${ }^{38-41,43,73,78,79,82,83}$ were identified as the main contributors to heterogeneity by using Galbraith plots (Supplementary Fig. 2). The heterogeneity was effectively decreased after excluding the outlier comparisons, and the SMD value and 95\% CI did not change substantially (SMD obtained from fixed-effects model: $-0.86 ; 95 \% \mathrm{CI}:-0.99$ to -0.73 ; $\mathrm{I}^{2}: 36.6 \%$; $\left.P_{\text {heterogeneity }}=0.041\right)$. Similar results were observed when subgroup analyses stratified by the assay technique or storage procedure showed a robust decrease in fasting AG levels of obese patients for each subgroup, and the exclusion of outlier studies did not change the significance of the results (Table 2).

Fasting DAG. The fasting circulation concentrations of des-acyl ghrelin were reported in 7 studies $^{40,41,65,74,75,78,80}$ with 360 obese patients and 179 healthy controls; meanwhile, 8 studies ${ }^{37,42,43,58,64,68,73,82}$ with 208 patients and 139 controls measured both blood acyl ghrelin and total ghrelin baseline levels, which were used to calculate the DAG levels. Pooled analysis showed that circulating fasting DAG levels were significantly decreased in obese patients compared with control subjects (SMD obtained from random-effects model: $-1.06 ; 95 \% \mathrm{CI}:-1.43$ to $\left.-0.69 ; P_{\mathrm{SMD}}<0.001\right)$, although the overall heterogeneity was apparent $\left(\mathrm{I}^{2}: 82.3 \%\right.$, $P_{\text {heterogeneity }}<0.001$ ) (Fig. 3 and Table 3). The Galbraith plot indicated that the assay results of 4 articles ${ }^{43,78,80,82}$ were largely responsible for this heterogeneity (Supplementary Fig. 3). Exclusion of these studies resulted in an SMD of -1.11 ( -1.29 to $-0.94 ; P_{\mathrm{SMD}}<0.001$; fixed-effects model $)$ with a significant decrease in heterogeneity $\left(\mathrm{I}^{2}\right.$ : $\left.32.0 \% ; P_{\text {heterogeneity }}=0.143\right)$. The results of our meta-analyses were also consistent in subgroup analyses regardless of outlier study inclusion or exclusion (Table 3).

Postprandial AG. Ten articles ${ }^{38,41,44,59,60,64,70,72,73,78}$ presented the data on postprandial concentrations of acyl ghrelin, although different measurement times were reported. According to the number of studies within each time stratification point, we selected 30,60 and 120 min to conduct the meta-analyses. After exclusion of the study by Zwirska-Korczala et al. ${ }^{64}$ due to the lack of statistical data of standard deviation and the other two articles ${ }^{38,78}$ due to high heterogeneity (Supplementary Fig. 4), 3, 5 and 5 studies were included to compare the circulating AG levels between obese subjects and controls at 30, 60 and 120 min postprandial, respectively (Table 4). Stratified analyses demonstrated significantly lower levels of postprandial AG in obese subjects at each time point with a fixed-effects model. The SMD values and 95\% CIs of $30 \mathrm{~min}$ postprandial (SMD: $-0.85 ; 95 \%$ CI: -1.18 to $-0.53 ; P_{\mathrm{SMD}}<0.001 ; \mathrm{I}^{2}: 0.0 \%, P_{\text {heterogeneity }}=0.577$ ), 60 min postprandial (SMD: $-1.00 ; 95 \%$ CI: -1.37 to $-0.63 ; P_{\mathrm{SMD}}<0.001 ; \mathrm{I}^{2}: 0.0 \%, P_{\text {heterogeneity }}=0.410$ ) and $120 \mathrm{~min}$ postprandial (SMD: $-1.21 ; 95 \% \mathrm{CI}:-1.59$ 


\begin{tabular}{|c|c|c|c|c|c|c|c|c|c|c|c|}
\hline References & Country & Group & \begin{tabular}{|l} 
Sample size \\
(male/female)
\end{tabular} & Age (year) & BMI $\left(\mathrm{kg} / \mathrm{m}^{2}\right)$ & Meal test & Out-come & Sampling time & Sample origin & $\begin{array}{l}\begin{array}{l}\text { Sample } \\
\text { procedure }\end{array} \\
\end{array}$ & Technique \\
\hline \multirow{2}{*}{ Baranowska $^{62}$} & \multirow{2}{*}{ Poland } & Control & $0 / 45$ & $26.0 \pm 7.6$ & $21.5 \pm 0.3$ & \multirow{2}{*}{ NA } & \multirow{2}{*}{ AG } & \multirow{2}{*}{ Fasting } & \multirow{2}{*}{ Plasma } & \multirow{2}{*}{ NR } & \multirow{2}{*}{ RIA } \\
\hline & & Obese & \begin{tabular}{|l|}
$0 / 37$ \\
\end{tabular} & $41.6 \pm 12.4$ & $32.7 \pm 0.8$ & & & & & & \\
\hline \multirow{2}{*}{ Homaee $^{39}$} & \multirow{2}{*}{ Iran } & Control & $19 / 0$ & $26.9 \pm 1.3$ & $18.5 \pm 0.5$ & \multirow{2}{*}{ NA } & \multirow{2}{*}{ AG } & \multirow{2}{*}{ Fasting } & \multirow{2}{*}{ Plasma } & \multirow{2}{*}{$\begin{array}{l}\text { EDTA, Aproti- } \\
\text { nine, HCL }\end{array}$} & FEISA \\
\hline & & Obese & $19 / 0$ & $27.5 \pm 1.3$ & $31.0 \pm 0.8$ & & & & & & LLAOT \\
\hline & & Control & $0 / 29$ & $37.0 \pm 2.0$ & $21.5 \pm 0.4$ & & & & & Parahydrox- & \\
\hline Iceta $^{\text {/S }}$ & France & Obese & $0 / 55$ & $38.0 \pm 1.5$ & $41.5 \pm 0.8$ & NA & AG, DAG & Fasting & Plasma & $\begin{array}{l}\text { ymercuriben- } \\
\text { zoic acid, HCL }\end{array}$ & ELISA \\
\hline$Y$ & Dolog & Control & $0 / 15$ & $42.9 \pm 5.3$ & $22.3 \pm 0.5$ & & & & & & \\
\hline NOIOCZIEjSKI & Poidia & Obese & $0 / 15$ & $42.2 \pm 3.3$ & $39.8 \pm 1.0$ & NA & AU, IS & rastng & serum & PIVIOS, HLL & NA \\
\hline Nakahara ${ }^{65}$ & Japan & Control & $0 / 11$ & $25.7 \pm 6.7$ & $21.8 \pm 3.1$ & NA & $A G, D A G$ & Fasting & Plasma & EDTA, Aproti- & ELISA \\
\hline Nandilaria & Japait & Obese & $0 / 10$ & $27.7 \pm 8.2$ & $28.4 \pm 2.7$ & NAn & | RU, & T & F Tasmina & nine, HCL & LEIOSA \\
\hline & & Control & $11 / 19$ & $44.0 \pm 2.0$ & $23.1 \pm 0.5$ & & & & & & \\
\hline Ezquerro $^{74}$ & Spain & Obese-NG & $16 / 28$ & $39.0 \pm 2.0$ & $46.4 \pm 1.3$ & NA & AG, DAG & Fasting & Plasma & NR & ELISA \\
\hline & & Obese-IGT & $17 / 25$ & $44.0 \pm 2.0$ & $43.2 \pm 1.0$ & & & & & & \\
\hline Holuzílon'69 & Crebr Penublis & Control & $0 / 15$ & $44.1 \pm 2.8$ & $22.2 \pm 0.5$ & NA & $\Delta C$ & Fosting & Serum & DPP-IV inhibi- & MILLIPLEX \\
\hline Haluzıкоva & Czecn Kepudic & Obese & \begin{tabular}{|l|}
$0 / 17$ \\
\end{tabular} & $39.9 \pm 2.0$ & $43.2 \pm 1.7$ & NA & AG & Fastung & serum & tor, Aprotinine & MAP \\
\hline Tambli42 & IUSA & Control & $0 / 9$ & $36.0 \pm 4.0$ & $22.0 \pm 1.0$ & NA & AC TC & Festing & Plasmp & ETDA, Aproti- & FUISA \\
\hline Tamloun & OSR & Obese & $0 / 9$ & $41.0 \pm 4.0$ & $44.0 \pm 2.0$ & NA & AU, IS & rasting & Prasma & nine, $\mathrm{HCl}$ & ELIOA \\
\hline S & IUSA & Control & $0 / 8$ & $44.2 \pm 5.5$ & $22.7 \pm 1.7$ & NA & I & Fosting & Plosme & Aprotinine, & RIA \\
\hline Savage & USA & Obese & \begin{tabular}{|l|}
$0 / 19$ \\
\end{tabular} & $36.5 \pm 1.5$ & $38.6 \pm 1.3$ & NA & AG & Fastung & Plasma & HCL & KIA \\
\hline A rafat68 & Germany & Control & $6 / 7$ & $25.1 \pm 0.6$ & $21.7 \pm 0.6$ & NA & $A C$ TC & Fasting & Plasma & NR & RIA \\
\hline Aratat & Germany & Obese & $5 / 6$ & $28.4 \pm 2.6$ & $34.4 \pm 1.7$ & N & No, 10 & Tasming & P Tasma & IN & Non \\
\hline & & Control & $25 / 30$ & $56.0 \pm 2.0$ & $23.1 \pm 0.3$ & & & & & & \\
\hline Rodríguez $^{40}$ & Spain & Obese-NG & $41 / 25$ & $55.0 \pm 1.0$ & $32.5 \pm 0.5$ & NA & AG, DAG & Fasting & Plasma & NR & ELISA \\
\hline & & Obese-IGT & $21 / 16$ & $59.0 \pm 1.0$ & $33.5 \pm 0.8$ & & & & & & \\
\hline Dunno7 & JSA & Control & $0 / 8$ & $40.0 \pm 3.2$ & $23.0 \pm 0.7$ & NI & $\Delta C$ & Fosting to & Dlocme & Aprotinine, & PIA \\
\hline Dentit & $00 \Omega$ & Obese & $0 / 14$ & $40.0 \pm 2.1$ & $40.0 \pm 1.3$ & NA & Hu & 1 asturg & F Tosina & HCL & THin \\
\hline & & Control & $5 / 12$ & NR & $22.3 \pm 0.5$ & & & & & EDTA, Aproti- & RIA \\
\hline Carroll $^{\circ 0}$ & USA & Obese & $12 / 22$ & NR & $43.4 \pm 0.9$ & NA & AG & Fasting & Plasma & $\begin{array}{l}\text { nine, DPP-IV } \\
\text { inhibitor }\end{array}$ & RIA \\
\hline Marzull 58 & Italy & Control & $10 / 10$ & $31.7 \pm 1.3$ & $22.4 \pm 0.6$ & Nat & $A C$ TC & Fosting & Plocme & FDTA HCI & RIA \\
\hline Niarzuno & Itaiy & Obese & $10 / 10$ & $32.4 \pm 1.6$ & $41.3 \pm 1.1$ & NN & RU, & I tasting & F Hosina & LDIR, IICL & Tin \\
\hline Suematsu 61 & Janan & Control & $16 / 1$ & $36.0 \pm 1.9$ & $22.2 \pm 0.6$ & NA & $A G$ & Fasting & Plasma & NR & BIA \\
\hline suematsu & Japan & Obese & $16 / 1$ & $35.5 \pm 1.8$ & $28.7 \pm 1.2$ & NA & AG & Fasting & Plasma & NK & KIA \\
\hline $\mathrm{Bik}^{63}$ & Poland & Control & $0 / 45$ & $26.0 \pm 7.6$ & $21.5 \pm 0.3$ & NA & $A G$ & Fasting & Plasme & Anrotinine & RIA \\
\hline DIK & Porana & Obese & $0 / 37$ & $31.6 \pm 8.2$ & $32.7 \pm 0.8$ & NA & AG & Fasting & Prasma & Aprotinme & KIA \\
\hline Marzullo $0^{37}$ & Italy & Control & 8 & NR & $22.1 \pm 1.2$ & NA & AG TG & Fasting & Plasma & NR & BIA \\
\hline Mrarzuno & Itary & Obese & 8 & NR & $33.7 \pm 1.5$ & NA & AG, IG & Fasting & Plasma & NR & RIA \\
\hline & & Control & 25 & NR & NR & & & & & & \\
\hline Yunker ${ }^{76}$ & USA & Over weight & 24 & NR & NR & NA & AG & Fasting & Plasma & NR & $\begin{array}{l}\text { MILLIPLEX } \\
\text { MAP }\end{array}$ \\
\hline & & Obese & 20 & NR & NR & & & & & & \\
\hline & & Control & $5 / 16$ & $33.0 \pm 1.3$ & $22.1 \pm 0.6$ & & & & & & \\
\hline Nogueira $^{80}$ & France & $\begin{array}{l}\text { Obese-low } \\
\text { HDL-c }\end{array}$ & $6 / 15$ & $34.0 \pm 2.0$ & $48.4 \pm 1.8$ & NA & DAG & Fasting & Serum & NR & ELISA \\
\hline & & Obese-MetS & $6 / 11$ & $38.0 \pm 2.7$ & $43.3 \pm 1.1$ & & & & & & \\
\hline & & Obese & $4 / 17$ & $37.0 \pm 1.1$ & $41.4 \pm 0.9$ & & & & & & \\
\hline & & Control & $24 / 26$ & $26.4 \pm 0.8$ & $22.7 \pm 0.2$ & & & & & \begin{tabular}{|l|} 
Parahydrox- \\
\end{tabular} & \\
\hline Lopez-Aguilar $^{77}$ & Mexico & Obese & $26 / 54$ & $29.2 \pm 0.70$ & $35.4 \pm 0.6$ & NA & AG & Fasting & Serum & $\begin{array}{l}\text { ymercuriben- } \\
\text { zoic acid, } \\
\text { EDTA, }\end{array}$ & ELISA \\
\hline & & Low weight & $16 / 15$ & $28.8 \pm 2.9$ & $17.6 \pm 0.1$ & & & & & & \\
\hline & & Normal weight & $14 / 14$ & $40.8 \pm 3.9$ & $21.7 \pm 0.3$ & & & & & & \\
\hline Ozkan $^{79}$ & Turkey & Over weight & $15 / 15$ & $52.2 \pm 2.3$ & $27.4 \pm 0.4$ & NA & AG & Fasting & Serum & Aprotinine & ELISA \\
\hline & & Obese & $16 / 15$ & $52.1 \pm 2.8$ & $34.9 \pm 0.4$ & & & & & & \\
\hline & & Morbidly obese & $15 / 15$ & $45.8 \pm 2.3$ & $44.8 \pm 0.8$ & & & & & & \\
\hline Coliconn 81 & IUSA & Control & $7 / 9$ & $33.0 \pm 1.6$ & $23.0 \pm 1.7$ & DIA & $A C$ & Feting & Plocmeng & EDTA, Apro- & FUUSA \\
\hline & & Morbidly obese & $9 / 12$ & $35.2 \pm 1.6$ & $49.4 \pm 5.3$ & NVIX & Tiv & f asung & 1 Hasinta & tinine, & DLIONA \\
\hline & & Control & $22 / 24$ & $51.2 \pm 1.0$ & $23.4 \pm 0.2$ & & & & & & \\
\hline Karcz-Socha ${ }^{82}$ & & $\begin{array}{l}\text { Moderately } \\
\text { obese }\end{array}$ & $21 / 22$ & $50.5 \pm 0.9$ & $32.5 \pm 0.2$ & NA & AG, TG & Fasting & Plasma & $\begin{array}{l}\text { EDTA, Aproti- } \\
\text { nine, DPP-IV } \\
\text { inhibito, PMSF }\end{array}$ & RIA \\
\hline & & Morbidly obese & $27 / 26$ & $52.3 \pm 0.9$ & $37.5 \pm 0.2$ & & & & & & \\
\hline
\end{tabular}




\begin{tabular}{|c|c|c|c|c|c|c|c|c|c|c|c|}
\hline References & Country & Group & \begin{tabular}{|l|}
$\begin{array}{l}\text { Sample size } \\
\text { (male/female) }\end{array}$ \\
\end{tabular} & Age (year) & BMI $\left(\mathrm{kg} / \mathrm{m}^{2}\right)$ & Meal test & Out-come & Sampling time & Sample origin & \begin{tabular}{|l|}
$\begin{array}{l}\text { Sample } \\
\text { procedure }\end{array}$ \\
\end{tabular} & Technique \\
\hline \multirow{3}{*}{$\begin{array}{l}\text { Krzyzanowska- } \\
\text { Swiniarska }^{83}\end{array}$} & \multirow{3}{*}{ Poland } & Control & $0 / 32$ & $28.8 \pm 0.8$ & $21.3 \pm 0.3$ & \multirow{3}{*}{ NA } & \multirow{3}{*}{ AG } & \multirow{3}{*}{ Fasting } & \multirow{3}{*}{ Serum } & \multirow{3}{*}{$\begin{array}{l}\text { Aprotinine, } \\
\text { HCL, PMSF }\end{array}$} & \multirow{3}{*}{ RIA } \\
\hline & & $\begin{array}{l}\begin{array}{l}\text { Obese without } \\
\text { insulin resist- } \\
\text { ance }\end{array} \\
\end{array}$ & $0 / 30$ & $32.5 \pm 1.2$ & $34.4 \pm 0.7$ & & & & & & \\
\hline & & $\begin{array}{l}\text { Obese with } \\
\text { insulin resist- } \\
\text { ance }\end{array}$ & $0 / 30$ & $32.3 \pm 1.2$ & $37.8 \pm 1.0$ & & & & & & \\
\hline \multirow{3}{*}{$\begin{array}{l}\text { Zwirska-Korc- } \\
\text { zala }^{64}\end{array}$} & \multirow{3}{*}{ Poland } & Control & $0 / 8$ & $33.9 \pm 3.7$ & $23.2 \pm 0.7$ & \multirow{3}{*}{$\begin{array}{l}\text { Standard mixed } \\
\text { breakfast } \\
(527 \mathrm{kcal})\end{array}$} & \multirow{3}{*}{ AG,TG } & \multirow{3}{*}{$\begin{array}{l}\text { Fasting and } \\
\text { postprandial } \\
30,60,120 \mathrm{~min}\end{array}$} & \multirow{3}{*}{ Plasma } & \multirow{3}{*}{$\begin{array}{l}\text { EDTA, PMSF, } \\
\text { HCL, DPP-IV } \\
\text { inhibitor }\end{array}$} & \multirow{3}{*}{ RIA } \\
\hline & & $\begin{array}{l}\begin{array}{l}\text { Moderately } \\
\text { obese-MetS }\end{array} \\
\end{array}$ & $0 / 12$ & $37.1 \pm 2.2$ & $34.9 \pm 0.9$ & & & & & & \\
\hline & & \begin{tabular}{|l|}
$\begin{array}{l}\text { Morbidly } \\
\text { obese-MetS }\end{array}$ \\
\end{tabular} & $0 / 17$ & $32.3 \pm 1.7$ & $46.9 \pm 1.6$ & & & & & & \\
\hline \multirow[b]{2}{*}{$\operatorname{Rizi}^{44}$} & \multirow[b]{2}{*}{ Singapore } & Control & $9 / 0$ & $23.2 \pm 0.2$ & $22 \pm 0.2$ & \multirow{2}{*}{$\begin{array}{l}\text { High-protein } \\
\text { test, high-fat } \\
\text { test, high- } \\
\text { carbohydrate } \\
\text { test (isocaloric } \\
600 \text { kcal) }\end{array}$} & \multirow[b]{2}{*}{ AG } & \multirow[b]{2}{*}{$\begin{array}{l}\text { Fasting and } \\
\text { postprandial } \\
30,60,90,120 \text {, } \\
180 \mathrm{~min}\end{array}$} & \multirow[b]{2}{*}{ Plasma } & \multirow[b]{2}{*}{$\begin{array}{l}\text { EDTA, DPP-IV } \\
\text { inhibitor, } \\
\text { Aprotinine }\end{array}$} & \multirow[b]{2}{*}{ ELISA } \\
\hline & & Obese & $9 / 0$ & $28.6 \pm 1.4$ & $30.1 \pm 0.7$ & & & & & & \\
\hline \multirow{2}{*}{ Brede $^{73}$} & \multirow{2}{*}{ Germany } & Control & $20 / 0$ & $24.1 \pm 3.7$ & $22.4 \pm 1.5$ & \multirow{2}{*}{$\begin{array}{l}\text { Ad libitum } \\
\text { test buffet } \\
\text { (1500 kcal) }\end{array}$} & \multirow{2}{*}{ AG,TG } & \multirow{2}{*}{\begin{tabular}{|l|}
$\begin{array}{l}\text { Fasting and } \\
\text { postprandial } 30 \\
\text { minites }\end{array}$ \\
\end{tabular}} & \multirow{2}{*}{ Plasma } & \multirow{2}{*}{ Aprotinine } & \\
\hline & & Obese & $20 / 0$ & $25.2 \pm 3.7$ & $34.9 \pm 3.6$ & & & & & & RIA \\
\hline & & Control & $10 / 10$ & $37.5 \pm 3.4$ & $22.4 \pm 0.3$ & Standard break- & & & & & \\
\hline Douglas $^{78}$ & UK & Obese & $12 / 11$ & $45 \pm 2.6$ & $29.2 \pm 0.6$ & $\begin{array}{l}\text { for males and } \\
513 \mathrm{kcal} \text { for } \\
\text { females) }\end{array}$ & AG, DAG & $\begin{array}{l}\text { Fastıng and } \\
\text { postprandial } \\
30,60,90 \mathrm{~min}\end{array}$ & Plasma & Aprotinine & ELISA \\
\hline & & Control & $20 / 0$ & $27.0 \pm 1.0$ & $22.0 \pm 0.3$ & Standard mixed & & Fasting and & & Parahydrox- & \\
\hline Seyssel $^{72}$ & Spain & Obese & $17 / 0$ & $29.0 \pm 2.0$ & $31.9 \pm 0.4$ & $\begin{array}{l}\text { breakfast } \\
(706 \mathrm{kcal})\end{array}$ & AG & $\begin{array}{l}\text { postprandial } 60 \\
\text { minites }\end{array}$ & Plasma & $\begin{array}{l}\text { ymercuriben- } \\
\text { zoic acid, } \\
\text { EDTA, } \mathrm{HCl}\end{array}$ & ELISA \\
\hline & & Control & $1 / 12$ & $37.2 \pm 2.6$ & $23.0 \pm 1.0$ & & & Fasting and & & EDTA, Par- & \\
\hline Dardzińska $^{41}$ & Poland & Obese & $7 / 17$ & $35.4 \pm 1.9$ & $43.8 \pm 0.7$ & $\begin{array}{l}\text { Mixed-meal } \\
\text { (300 kcal) }\end{array}$ & AG, DAG & $\begin{array}{l}\text { postprandial } \\
120 \text { minites }\end{array}$ & Plasma & $\begin{array}{l}\text { ahydroxymer- } \\
\text { curibenzoic } \\
\text { acid }\end{array}$ & ELISA \\
\hline & & Control & 14 & $26 \pm 1.6$ & $22.9 \pm 0.5$ & & & Fasting and & & & \\
\hline Heden $^{70}$ & USA & Obese & 14 & $25.1 \pm 1.3$ & $34.8 \pm 1.2$ & $\begin{array}{l}\text { Mixed meal } \\
(600 \mathrm{kcal})\end{array}$ & AG & $\begin{array}{l}5,10,15,20 \\
30,40,50,60 \\
75,90,120 \\
150,180,210 \\
240 \mathrm{~min}\end{array}$ & Plasma & $\begin{array}{l}\text { EDTA, Apro- } \\
\text { tinine }\end{array}$ & $\begin{array}{l}\text { MILLIPLEX } \\
\text { MAP }\end{array}$ \\
\hline & & Control & $7 / 0$ & $22.4 \pm 1.6$ & $22.4 \pm 0.9$ & & & Fasting and & & & \\
\hline $\mathrm{Ueda}^{38}$ & Japan & Obese & $7 / 0$ & $22.9 \pm 1.3$ & $30 \pm 1.2$ & $\begin{array}{l}\text { Standard break- } \\
\text { fast (560 kcal) }\end{array}$ & AG & $\begin{array}{l}\text { postprandial } \\
60,90,120 \\
150,180 \mathrm{~min}\end{array}$ & Plasma & $\begin{array}{l}\text { EDTA, Apro- } \\
\text { tinine }\end{array}$ & ELISA \\
\hline & & Control & $0 / 8$ & $40.2 \pm 4.0$ & $21.8 \pm 0.8$ & Carbohydrate- & & Fasting and & & & \\
\hline Tentolouris $^{59}$ & Greece & Obese & $0 / 8$ & $39.9 \pm 5.3$ & $35.51 \pm 1.6$ & $\begin{array}{l}(546 \mathrm{kcal}) \text {, } \\
\text { fat-rich meal } \\
(532 \mathrm{kcal})\end{array}$ & AG & $\begin{array}{l}\text { postprandial } \\
60,120 \\
180 \mathrm{~min}\end{array}$ & Plasma & NR & RIA \\
\hline & & Control & $3 / 3$ & $26.2 \pm 0.7$ & $22.5 \pm 0.7$ & & & Fasting and & & & \\
\hline Foschi $^{60}$ & Italy & Obese & $1 / 11$ & $41.1 \pm 3.8$ & $42.9 \pm 1.3$ & $\begin{array}{l}\text { Liquid test meal } \\
\text { (504 kcal) }\end{array}$ & AG & \begin{tabular}{|l} 
postprandial \\
60,120 \\
$180 \mathrm{~min}$ \\
\end{tabular} & Plasma & EDTA & RIA \\
\hline
\end{tabular}

Table 1. Summary of general characteristics of the included studies. Age and BMI are presented as the means \pm SEM; MetS metabolic syndrome, $H D L-c$ high-density lipoprotein-cholesterol, $N G$ normoglycemia, $I G T$ impaired glucose tolerance, $A G$ acyl ghrelin, $D A G$ des-acyl ghrelin, ELISA enzyme-linked immunosorbent assay, RIA radioimmunoassay, MILLIPLEX MAP magnetic bead-based quantitative multiplex immunoassay, EDTA ethylene diamine tetraacetic acid, $P M S F$ phenylmethylsulfonyl fluoride, $H C L$ hydrogen chloride, NA not applicable, $N R$ not reported.

to $\left.-0.83 ; P_{\mathrm{SMD}}<0.001 ; \mathrm{I}^{2}: 42.2 \%, P_{\text {heterogeneity }}=0.140\right)$ did not change substantially after excluding the outlier comparison (Fig. 4 and Table 4 ).

Changes in postprandial AG. For healthy individuals, stratification by time points demonstrated a statistically significant decrease in blood AG levels in the $30 \mathrm{~min}$ (SMD obtained from random-effects model: -0.85 ; 95\%CI: -1.48 to $\left.-0.21 ; P_{\mathrm{SMD}}=0.009 ; \mathrm{I}^{2}: 77.5 \% ; P_{\text {heterogeneity }}=0.004\right), 60 \mathrm{~min}$ (SMD obtained from fixed-effects model: -0.72 ; $95 \% \mathrm{CI}:-1.02$ to $\left.-0.41 ; P_{\mathrm{SMD}}<0.001 ; \mathrm{I}^{2}: 25.7 \% ; P_{\text {heterogeneity }}=0.233\right)$ and $120 \mathrm{~min}(\mathrm{SMD}$ obtained from fixed-effects model: -0.42 ; $95 \% \mathrm{CI}:-0.77$ to $\left.-0.06 ; P_{\mathrm{SMD}}=0.021 ; \mathrm{I}^{2}: 16.8 \% ; P_{\text {heterogeneity }}=0.305\right)$ following meal test intervention compared with fasting states (Fig. 5 and Table 5). A high level of heterogeneity can be observed in the stratification of $30 \mathrm{~min}$, and the study by E. P. Rizi et al. ${ }^{44}$ was considered the main cause of the heterogeneity via the Galbraith plot (Supplementary Fig. 5). With the exclusion of this article, heterogeneity decreased significantly $\left(\mathrm{I}^{2}: 0.0 \% ; P_{\text {heterogeneity }}=0.371\right)$, and the significance of the result remained consistent (SMD obtained from the fixed-effects model: $-1.19 ; 95 \% \mathrm{CI}:-1.54$ to $\left.-0.84 ; P_{\mathrm{SMD}}<0.001\right)$.

For obese individuals, stratification by time points demonstrated a statistically significant decrease in blood AG levels in the $30 \mathrm{~min}$ (SMD obtained from fixed-effects model: $-0.61 ; 95 \% \mathrm{CI}:-0.89$ to $-0.34 ; P_{\mathrm{SMD}}<0.001$; $\mathrm{I}^{2}: 0.0 \% ; P_{\text {heterogeneity }}=0.762$ ) and $60 \mathrm{~min}$ (SMD obtained from fixed-effects model: $-0.62 ; 95 \% \mathrm{CI}:-0.91$ to -0.33 ; 


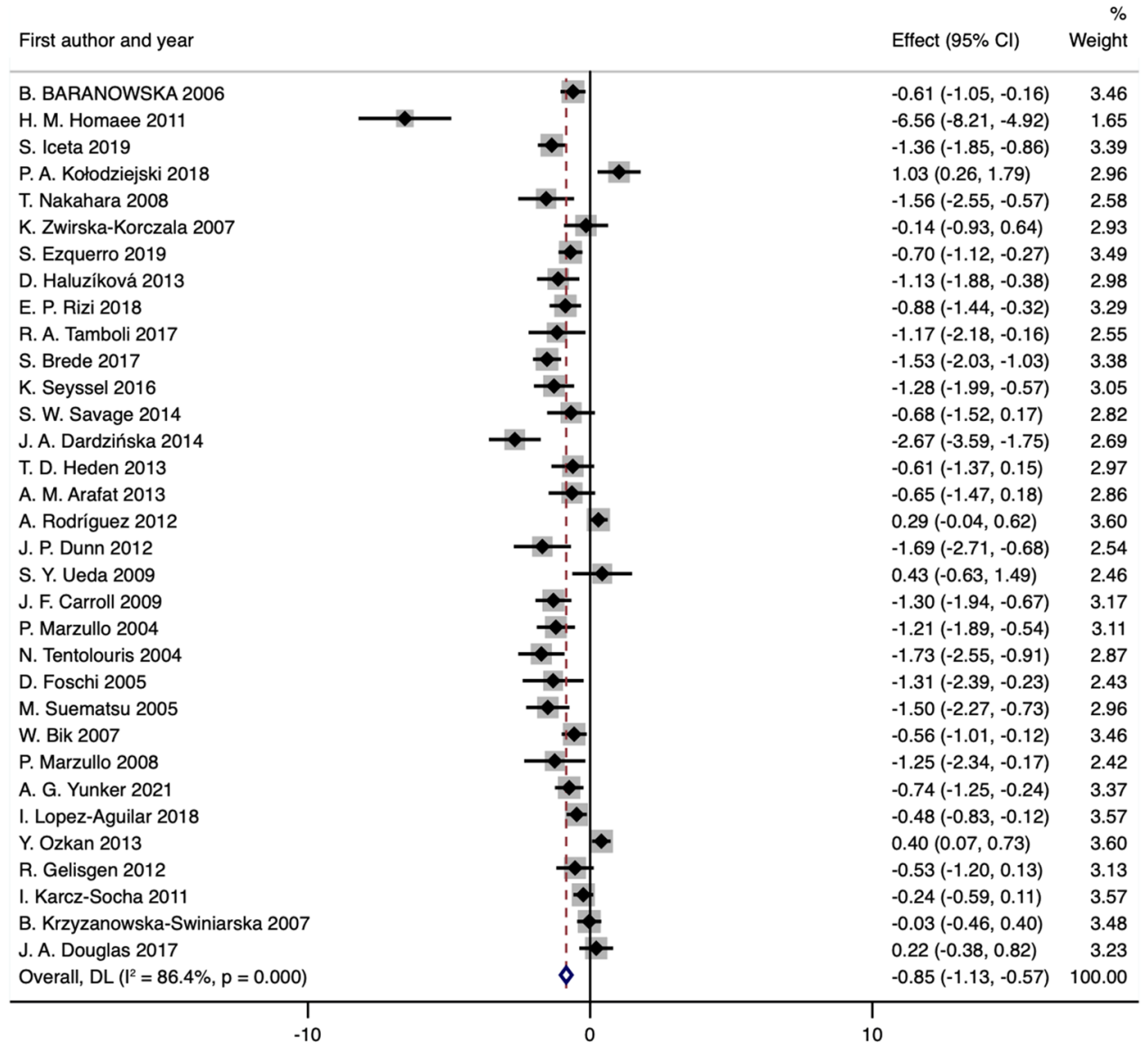

NOTE: Weights are from random-effects model

Figure 2. Forest plot for comparisons of fasting AG levels (obesity vs. normal weight).

$\left.P_{\mathrm{SMD}}<0.001 ; \mathrm{I}^{2}: 15.9 \% ; P_{\text {heterogeneity }}=0.309\right)$ following meal test intervention, but there was no significant difference between postprandial $120 \mathrm{~min}$ and baseline states (SMD obtained from fixed-effects model: $-0.28 ; 95 \%$ CI: -0.60 to $\left.0.03 ; P_{\mathrm{SMD}}=0.074 ; \mathrm{I}^{2}: 24.0 \% ; P_{\text {heterogeneity }}=0.254\right)($ Fig. 6 and Table 5$)$.

The mean change of AG concentration (calculated as the differential between baseline and postprandial states) was similar in both obese and lean health groups at each time point $\left(\Delta \mathrm{SMD}_{30 \mathrm{~min}}: 0.31,95 \% \mathrm{CI}:-0.35\right.$ to $0.97, P_{\mathrm{SMD}}=0.359, \mathrm{I}^{2}: 81.0 \% ; P_{\text {heterogeneity }}=0.001 ; \Delta \mathrm{SMD}_{60 \mathrm{~min}}: 0.17,95 \% \mathrm{CI}:-0.12$ to $0.46, P_{\mathrm{SMD}}=0.246, \mathrm{I}^{2}: 0.0 \%$; $P_{\text {heterogeneity }}=0.920 ; \Delta \mathrm{SMD}_{120 \mathrm{~min}}: 0.21,95 \% \mathrm{CI}:-0.13$ to $0.54, P_{\mathrm{SMD}}=0.224, \mathrm{I}^{2}: 0.0 \% ; P_{\text {heterogeneity }}=0.884$, randomeffects model, Fig. 7 and Table 6), even the exclusion of the study by S. Brede et al. ${ }^{73}$ in the stratification of $30 \mathrm{~min}$ for heterogeneity (Supplementary Fig. 6), the results of our meta-analyses remained consistent $\left(\triangle \mathrm{SMD}_{30 \mathrm{~min}}: 0.03\right.$, $95 \%$ CI: -0.38 to $0.33, P_{\mathrm{SMD}}=0.887, \mathrm{I}^{2}: 0.0 \% ; P_{\text {heterogeneity }}=0.541$, Table 6$)$.

Postprandial DAG. Only four included studies ${ }^{41,64,73,78}$ reported postprandial TG or DAG levels, and one of them was excluded because of the lack of standard deviation data for the DAG calculation. The remaining three studies investigated peripheral blood hormones after the meal test, but time points were inconsistent and were not suitable for a meta-analysis, as such, this postprandial DAG group was not considered further.

Publication bias. The results of Egger's and Begg's tests detected that there might be a publication bias for the outcome of fasting AG levels ( $\operatorname{Pr}>|z|=0.010$ for Begg's test and $P>|t|=0.000$ for Egger's test) (Supplementary Fig. 7). To clarify this problem, a trim-and-fill method was used to adjust the results, no trimming was performed, and the data were unchanged. There was no publication bias in the literature, and the significant $P$ value of Begg's and Egger's tests may originate from other factors, such as mixed age, gender or ethnicity, in some studies. No publication bias was detected in the fasting DAG analysis $(\operatorname{Pr}>|z|=0.843$ for Begg's test and $P>|t|=0.792$ for Egger's test) (Supplementary Fig. 8).

\section{Discussion}

Ghrelin, an endogenous ligand of the GHSR, is the only known orexigenic gut hormone that increases appetite and food reward ${ }^{11,45}$. Although AG and DAG were described separately since ghrelin was first introduced in 1999 (Kojima et al. ${ }^{11}$ ), previous studies preferred to examine total plasma ghrelin without distinguishing AG and 


\begin{tabular}{|c|c|c|c|c|c|c|c|c|}
\hline \multirow{2}{*}{$\begin{array}{l}\text { Groups or } \\
\text { subgroups }\end{array}$} & \multirow[b]{2}{*}{$\mathbf{N}$} & \multirow[b]{2}{*}{ References } & \multicolumn{2}{|c|}{ Random-effects model } & \multicolumn{2}{|c|}{ Fix-effects model } & \multirow[b]{2}{*}{$I^{2}(\%)$} & \multirow[b]{2}{*}{$\boldsymbol{P}_{\text {Heterogeneit? }}$} \\
\hline & & & SMD $(95 \% \mathrm{CI})$ & $P_{\text {SMD }}$ & SMD (95\%CI) & $P_{\mathrm{SMD}}$ & & \\
\hline \multicolumn{9}{|l|}{ Fasting AG } \\
\hline All & 33 & $37-44,58-79,81-83$ & $\begin{array}{l}-0.85(-1.13 \\
\text { to }-0.57)\end{array}$ & $<0.001$ & $\begin{array}{l}-0.55(-0.65 \\
\text { to }-0.45)\end{array}$ & $<0.001$ & 86.4 & $<0.001$ \\
\hline \multicolumn{9}{|c|}{ Subgroup 1 technique } \\
\hline ELISA & 14 & $38-42,44,65,72,74,75,77-79,81$ & $\begin{array}{l}-0.95(-1.47 \\
\text { to }-0.43)\end{array}$ & $<0.001$ & $\begin{array}{l}-0.41(-0.55 \\
\text { to }-0.27)\end{array}$ & $<0.001$ & 91.7 & $<0.001$ \\
\hline RIA & 16 & $37,58-64,66-68,71,82,83$ & $\begin{array}{l}-0.80(-1.14 \\
\text { to }-0.45)\end{array}$ & $<0.001$ & $\begin{array}{l}-0.66(-0.81 \\
\text { to }-0.51)\end{array}$ & $<0.001$ & 78.5 & $<0.001$ \\
\hline MILLIPLEX MAP & 3 & $69,70,76$ & $\begin{array}{l}-0.81(-1.17 \\
\text { to }-0.44)\end{array}$ & $<0.001$ & $\begin{array}{l}-0.81(-1.17 \\
\text { to }-0.44)\end{array}$ & $<0.001$ & 0.0 & 0.596 \\
\hline \multicolumn{9}{|c|}{ Subgroup 2 enzymatic inhibitors contained } \\
\hline YES & 25 & $37-40,58-74,76-79,81-83$ & \begin{tabular}{|l|}
$-0.87(-1.22$ \\
to -0.53$)$
\end{tabular} & $<0.001$ & $\begin{array}{l}-0.57(-0.68 \\
\text { to }-0.46)\end{array}$ & $<0.001$ & 87.5 & $<0.001$ \\
\hline NO & 8 & $37,40,59,61,62,68,74,76$ & $\begin{array}{l}-0.79(-1.28 \\
\text { to }-0.31)\end{array}$ & 0.001 & $\begin{array}{l}-0.49(-0.67 \\
\text { to }-0.30)\end{array}$ & $<0.001$ & 83.1 & $<0.001$ \\
\hline \multicolumn{9}{|c|}{ Fasting AG after excluding the studies with heterogeneity } \\
\hline All & 23 & $37,42,44,58-72,74-77,81$ & \begin{tabular}{|l|}
$-0.92(-1.09$ \\
to -0.75$)$
\end{tabular} & $<0.001$ & $\begin{array}{l}-0.86(-0.99 \\
\text { to }-0.73)\end{array}$ & $<0.001$ & 36.6 & 0.041 \\
\hline \multicolumn{9}{|c|}{ Subgroup 1 technique } \\
\hline ELISA & 8 & $42,44,65,72,7,7,75,77,81$ & \begin{tabular}{|l|}
$-0.91(-1.19$ \\
to -0.62$)$ \\
\end{tabular} & $<0.001$ & $\begin{array}{l}-0.83(-1.03 \\
\text { to }-0.64)\end{array}$ & $<0.001$ & 47.6 & 0.064 \\
\hline RIA & 12 & $37,58-64,66-68,71$ & $\begin{array}{l}-0.98(-1.27 \\
\text { to }-0.70)\end{array}$ & $<0.001$ & $\begin{array}{l}-0.91(-1.11 \\
\text { to }-0.71)\end{array}$ & $<0.001$ & 44.9 & 0.046 \\
\hline MILLIPLEX MAP & 3 & $69,70,76$ & $\begin{array}{l}-0.81(-1.17 \\
\text { to }-0.44)\end{array}$ & $<0.001$ & $\begin{array}{l}-0.81(-1.17 \\
\text { to }-0.44)\end{array}$ & $<0.001$ & 0.0 & 0.596 \\
\hline \multicolumn{9}{|c|}{ Subgroup 2 enzymatic inhibitors contained } \\
\hline YES & 16 & $42,44,58,60,63-67,69-72,75,77,81$ & $\begin{array}{l}-0.93(-1.15 \\
\text { to }-0.71)\end{array}$ & $<0.001$ & $\begin{array}{l}-0.87(-1.03 \\
\text { to }-0.71)\end{array}$ & $<0.001$ & 40.0 & 0.050 \\
\hline NO & 7 & $37,59,61,62,68,74,76$ & $\begin{array}{l}-0.91(-1.22 \\
\text { to }-0.61)\end{array}$ & $<0.001$ & $\begin{array}{l}-0.85(-1.07 \\
\text { to }-0.62)\end{array}$ & $<0.001$ & 38.6 & 0.134 \\
\hline
\end{tabular}

Table 2. Meta-analysis for comparison of fasting AG levels (obesity vs. normal weight). AG acyl ghrelin, ELISA enzyme-linked-immunosorbent-assay, RIA radio-immuno-assay, MILLIPLEX MAP magnetic beadbased quantitative multiplex immunoassay, $N$ number of studies.

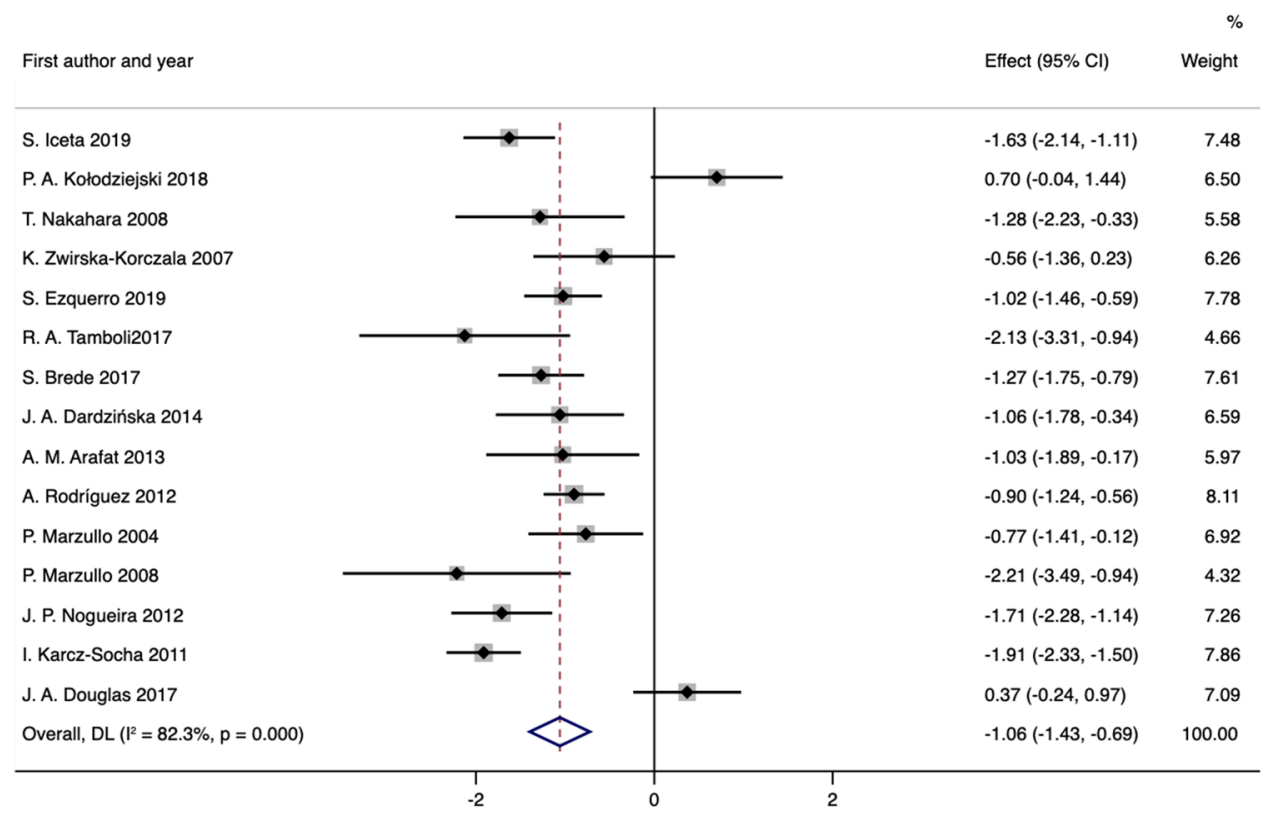

NOTE: Weights are from random-effects model

Figure 3. Forest plot for comparisons of fasting DAG levels (obesity vs. normal weight). 


\begin{tabular}{|c|c|c|c|c|c|c|c|c|}
\hline \multirow{2}{*}{$\begin{array}{l}\text { Groups or } \\
\text { subgroups }\end{array}$} & \multirow[b]{2}{*}{$\mathbf{N}$} & \multirow[b]{2}{*}{ References } & \multicolumn{2}{|c|}{ Random-effects model } & \multicolumn{2}{|c|}{ Fix-effects model } & \multirow[b]{2}{*}{$I^{2}(\%)$} & \multirow[b]{2}{*}{$\boldsymbol{P}_{\text {Heterogeneity }}$} \\
\hline & & & SMD $(95 \% \mathrm{CI})$ & $P_{\text {SMD }}$ & SMD $(95 \% \mathrm{CI})$ & $P_{\text {SMD }}$ & & \\
\hline \multicolumn{9}{|c|}{ Fasting DAG } \\
\hline All & 15 & $37,40-43,58,64,65,68,73-75,78,80,82$ & \begin{tabular}{|l}
$-1.06(-1.43$ \\
to -0.69$)$
\end{tabular} & $<0.001$ & \begin{tabular}{|l|}
$-1.09(-1.24$ \\
to -0.94$)$
\end{tabular} & $<0.001$ & 82.3 & $<0.001$ \\
\hline \multicolumn{9}{|c|}{ Subgroup 1 technique } \\
\hline ELISA & 8 & $40-42,65,74,75,7,8,80$ & $\begin{array}{l}-1.12(-1.58 \\
\text { to }-0.65)\end{array}$ & $<0.001$ & \begin{tabular}{|l|}
$-1.16(-1.39$ \\
to -0.92$)$
\end{tabular} & $<0.001$ & 86.2 & $<0.001$ \\
\hline RIA & 7 & $37,43,58,64,68,73,82$ & $\begin{array}{l}-0.98(-1.66 \\
\text { to }-0.31)\end{array}$ & 0.004 & $\begin{array}{l}-1.05(-1.24 \\
\text { to }-0.86)\end{array}$ & $<0.001$ & 80.2 & $<0.001$ \\
\hline \multicolumn{9}{|c|}{ Subgroup 2 enzymatic inhibitors contained } \\
\hline YES & 10 & $41-43,58,64,65,73,75,78,82$ & $\begin{array}{l}-0.94(-1.51 \\
\text { to }-0.37)\end{array}$ & 0.001 & \begin{tabular}{|l|}
$-1.07(-1.27$ \\
to -0.88$)$
\end{tabular} & $<0.001$ & 87.2 & $<0.001$ \\
\hline NO & 5 & $37,40,68,74,80$ & $\begin{array}{l}-1.22(-1.61 \\
\text { to }-0.84)\end{array}$ & $<0.001$ & $\begin{array}{l}-1.12(-1.35 \\
\text { to }-0.89)\end{array}$ & $<0.001$ & 54.7 & 0.066 \\
\hline \multicolumn{9}{|c|}{ Fasting DAG after excluding the studies with heterogeneity } \\
\hline All & 11 & $37,40-42,58,64,65,68,73-75$ & \begin{tabular}{|l}
$-1.14(-1.38$ \\
to -0.91$)$
\end{tabular} & $<0.001$ & \begin{tabular}{|l|}
$-1.11(-1.29$ \\
to -0.94$)$
\end{tabular} & $<0.001$ & 32.0 & 0.143 \\
\hline \multicolumn{9}{|c|}{ Subgroup 1 technique } \\
\hline ELISA & 6 & $40-42,65,74,75$ & \begin{tabular}{|l}
$-1.20(-1.51$ \\
to -0.89$)$
\end{tabular} & $<0.001$ & \begin{tabular}{|l|}
$-1.14(-1.35$ \\
to -0.92$)$
\end{tabular} & $<0.001$ & 40.6 & 0.135 \\
\hline RIA & 5 & $37,58,64,68,73$ & \begin{tabular}{|l|}
$-1.06(-1.47$ \\
to -0.65$)$
\end{tabular} & $<0.001$ & \begin{tabular}{|l|}
$-1.07(-1.38$ \\
to -0.76$)$
\end{tabular} & $<0.001$ & 35.1 & 0.187 \\
\hline \multicolumn{9}{|c|}{ Subgroup 2 enzymatic inhibitors contained } \\
\hline YES & 7 & $41,42,58,64,65,73,75$ & \begin{tabular}{|l}
$-1.21(-1.53$ \\
to -0.88$)$
\end{tabular} & $<0.001$ & \begin{tabular}{|l}
$-1.22(-1.47$ \\
to -0.97$)$
\end{tabular} & $<0.001$ & 36.5 & 0.150 \\
\hline NO & 4 & $37,40,68,74$ & $\begin{array}{l}-1.03(-1.35 \\
\text { to }-0.72)\end{array}$ & $<0.001$ & $\begin{array}{l}-1.00(-1.26 \\
\text { to }-0.75)\end{array}$ & $<0.001$ & 21.3 & 0.282 \\
\hline
\end{tabular}

Table 3. Meta-analysis for comparison of fasting DAG levels (obesity vs. normal weight). DAG des-acyl ghrelin, ELISA enzyme-linked-immunosorbent-assay, RIA radio-immuno-assay, MILLIPLEX MAP magnetic bead-based quantitative multiplex immunoassay, $N$ number of studies.

\begin{tabular}{|c|c|c|c|c|c|c|c|c|}
\hline \multirow[b]{2}{*}{ Groups or subgroups } & \multirow[b]{2}{*}{$\mathbf{N}$} & \multirow[b]{2}{*}{ References } & \multicolumn{2}{|l|}{ Random-effects model } & \multicolumn{4}{|c|}{ Fix-effects model } \\
\hline & & & SMD $(95 \% \mathrm{CI})$ & $P_{\text {SMD }}$ & SMD $(95 \% \mathrm{CI})$ & $P_{\mathrm{SMD}}$ & $I^{2}(\%)$ & $\boldsymbol{P}_{\text {Heterogeneity }}$ \\
\hline \multicolumn{9}{|c|}{ Postprandial AG stratified by duration of postprandial period } \\
\hline $30 \mathrm{~min}$ & 4 & $44,70,73,78$ & \begin{tabular}{|l}
$-0.60(-1.07$ \\
to -0.13$)$
\end{tabular} & 0.013 & \begin{tabular}{|l}
$-0.65(-0.93$ \\
to -0.36$)$
\end{tabular} & $<0.001$ & 62.0 & 0.048 \\
\hline $60 \mathrm{~min}$ & 7 & $38,44,59,60,70,72,78$ & $-0.57(-1.17$ to 0.02$)$ & 0.026 & $\begin{array}{l}-0.57(-0.87 \\
\text { to }-0.27)\end{array}$ & $<0.001$ & 72.8 & 0.001 \\
\hline $120 \mathrm{~min}$ & 6 & $38,41,44,59,60,70$ & $\begin{array}{l}-0.94(-1.59 \\
\text { to }-0.28)\end{array}$ & 0.005 & $\begin{array}{l}-1.01(-1.37 \\
\text { to }-0.65)\end{array}$ & $<0.001$ & 68.7 & 0.007 \\
\hline \multicolumn{9}{|c|}{ Postprandial AG stratified by duration of postprandial period after excluding the studies with heterogeneity } \\
\hline $30 \mathrm{~min}$ & 3 & $44,70,73$ & \begin{tabular}{|l}
$-0.85(-1.18$ \\
to -0.53$)$
\end{tabular} & $<0.001$ & \begin{tabular}{|l}
$-0.85(-1.18$ \\
to -0.53$)$
\end{tabular} & $<0.001$ & 0.0 & 0.577 \\
\hline $60 \mathrm{~min}$ & 5 & $44,59,60,70,72$ & $\begin{array}{l}-1.00(-1.37 \\
\text { to }-0.63)\end{array}$ & $<0.001$ & $\begin{array}{l}-1.00(-1.37 \\
\text { to }-0.63)\end{array}$ & $<0.001$ & 0.0 & 0.410 \\
\hline $120 \mathrm{~min}$ & 5 & $41,44,59,60,70$ & $\begin{array}{l}-1.20(-1.71 \\
\text { to }-0.69)\end{array}$ & $<0.001$ & $\begin{array}{l}-1.21(-1.59 \\
\text { to }-0.83)\end{array}$ & $<0.001$ & 42.2 & 0.140 \\
\hline
\end{tabular}

Table 4. Meta-analysis for comparison of postprandial AG levels stratified by duration of postprandial period (obesity vs. normal weight). $A G$ acyl ghrelin.

DAG before the theory that DAG may have independent actions suggested by Broglio et al. in $2004^{22}$. With the swiftly rising prevalence of obesity, it is important to evaluate appetite-related hormones precisely, the differences in which could both inform mechanisms in obesity and offer potential new pharmacological interventions.

To the best of our knowledge, this was the first systematic review and meta-analysis to compare the concentrations of AG and DAG separately between obese patients and healthy individuals while also considering the dietary states that can affect ghrelin levels. The main findings were that under a fasting state, both AG and DAG decreased significantly in obese groups compared with controls; for the postprandial state, a similar extent of AG decline can be observed in both groups, and a shorter duration of suppression existed in obese groups. 


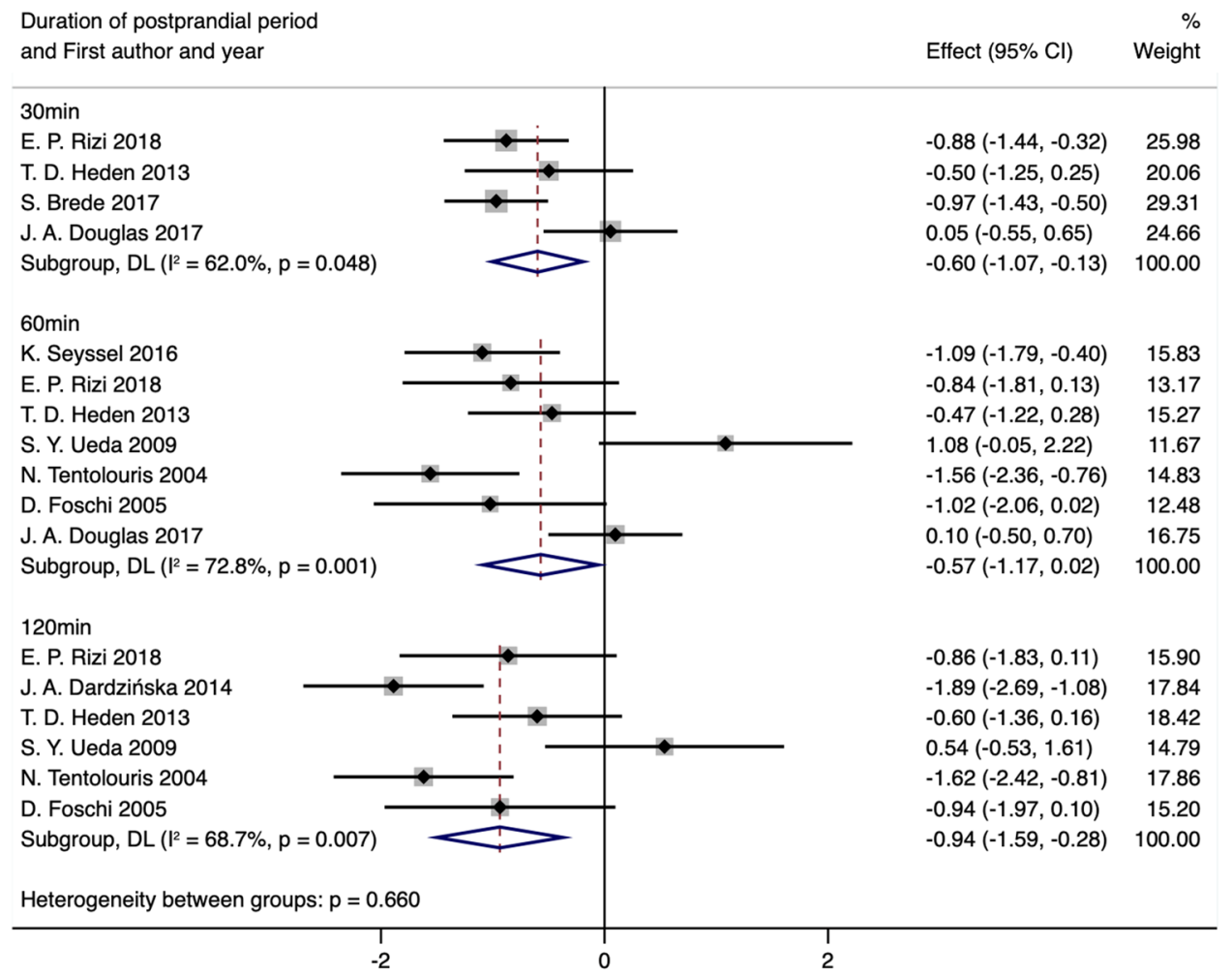

NOTE: Weights and between-subgroup heterogeneity test are from random-effects model

Figure 4. Forest plot for comparisons of postprandial AG levels stratified by duration of postprandial period (obesity vs. normal weight).

Fasting acyl and des-acyl ghrelin in obesity. Several studies have reported that obese individuals have higher fasting levels of circulating acyl ghrelin than lean subjects ${ }^{38,40,43}$, indicating that AG may play a key role in the cause of obesity directly or indirectly via stimulation of food intake. However, according to this meta-analysis, we demonstrated a reduction in circulating basal AG levels in obese adults (Fig. 2 and Table 2). Significant heterogeneity did exist, and after excluding the outlier studies that were identified by the Galbraith plots, the significance of the result remained virtually unchanged (Table 2). Similar reductions were also observed in the obese patients when circulating fasting DAG levels were pooled (Fig. 3 and Table 3). The simultaneous variation of AG and DAG can be partially explained by the common sense that esterase-catalyzed deacylation produces DAG from AG, and after intravenous injection, AG appears to induce the secretion of DAG in humans ${ }^{84}$. In addition, the reacylation of DAG to AG by the catalysis of plasma membrane-exposed GOAT has been proposed ${ }^{85}$.

The significant drop in both AG and DAG supports the hypothesis of physically compensatory adaptation, which aims to reduce a hunger stimulus by lowering plasma ghrelin concentrations under an energy surplus ${ }^{31}$, and the same phenomenon has been observed in people with binge eating ${ }^{86,87}$. The complicated ghrelin-GHSR system involves diverse hormonal signals, including gastrointestinal hormones, pancreatic hormones and multiple endocrine hormones ${ }^{88}$. Among the compensatory adaptations, the impact of glucose metabolism on energy homeostasis is well established. As a signal of positive energy, the increase in blood glucose stimulates the secretion of insulin and further suppresses ghrelin secretion, thus reducing plasma ghrelin levels ${ }^{89,90}$. In addition, recent studies have indicated that a positive energy balance impairs ghrelin's functions in homeostatic feeding and reward processing, leading to a condition called ghrelin resistance, which reduces ghrelin action in the brain ${ }^{26,91}$. Based on the attenuated metabolic sensitivity, it is not surprising that the intervention of additional reduction or suppression of ghrelin provides limited efficacy. Moreover, the disruption of energy homeostasis in the higher body weight set-point may result in a compensatory increase in newly synthesized ghrelin, to say nothing of side effects relevant to glycemic control, accounting for prospects in animal experiments upon short-term use of ghrelin or GHSR antagonism, while long-term clinical efficacy has been minimal ${ }^{49}$.

Given the methodological differences in assay techniques or storage procedures, which are critical for the extremely susceptible ester bond of AG in the circulation ${ }^{34}$, subgroup analyses were conducted and showed robust decreased basal AG and DAG levels in obese patients compared with lean subjects for each stratification stratified by either the assay technique or storage procedure (Tables 2 and 3). According to the commercial recommendations, Acidification, low temperature, and enzymatic inhibitors were indispensable. Although we failed to detect the difference under different sample processing, the subgroup analyses illustrated the stable reduction of both AG and DAG in obesity. 


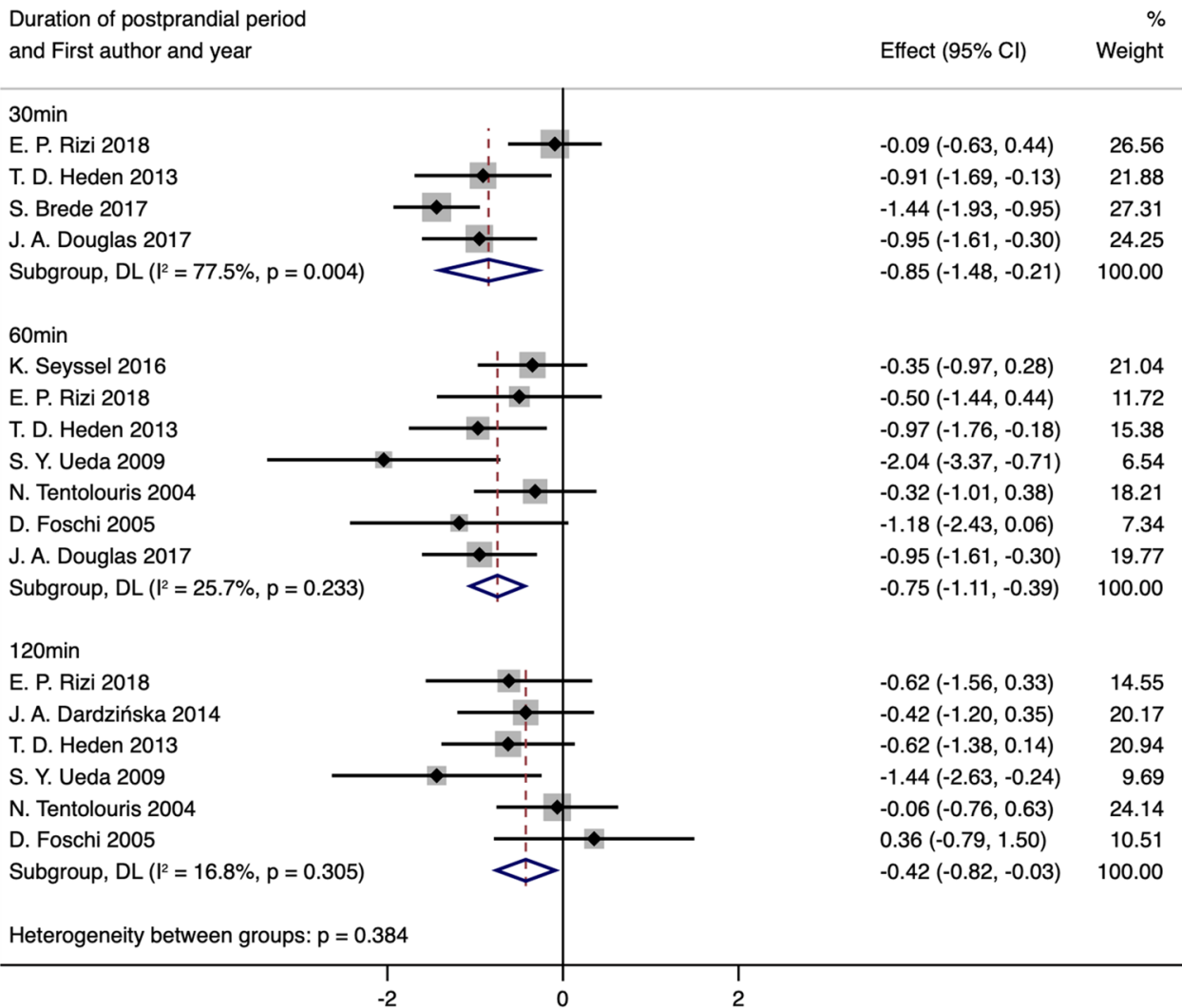

NOTE: Weights and between-subgroup heterogeneity test are from random-effects model

Figure 5. Forest plot for comparisons of postprandial AG levels stratified by duration of postprandial period in normal-weight group (postprandial vs. baseline).

\begin{tabular}{|c|c|c|c|c|c|c|c|c|}
\hline \multirow[b]{2}{*}{ Groups or subgroups } & \multirow[b]{2}{*}{$\mathbf{N}$} & \multirow[b]{2}{*}{ References } & \multicolumn{2}{|c|}{ Random-effects model } & \multicolumn{2}{|c|}{ Fix-effects model } & \multirow[b]{2}{*}{$I^{2}(\%)$} & \multirow[b]{2}{*}{$\boldsymbol{P}_{\text {Heterogeneity }}$} \\
\hline & & & SMD (95\%CI) & $P_{\text {SMD }}$ & SMD $(95 \% \mathrm{CI})$ & $P_{\text {SMD }}$ & & \\
\hline \multicolumn{9}{|c|}{ Postprandial AG stratified by duration of postprandial period in normal-weight group } \\
\hline $30 \mathrm{~min}$ & 4 & $44,70,73,78$ & $\begin{array}{l}-0.85(-1.48 \\
\text { to }-0.21)\end{array}$ & 0.009 & $\begin{array}{l}-0.86(-1.15 \\
\text { to }-0.56)\end{array}$ & $<0.001$ & 77.5 & 0.004 \\
\hline $60 \mathrm{~min}$ & 7 & $38,44,59,60,70,72,78$ & $\begin{array}{l}-0.75(-1.11 \\
\text { to }-0.39)\end{array}$ & $<0.001$ & $\begin{array}{l}-0.72(-1.02 \\
\text { to }-0.41)\end{array}$ & $<0.001$ & 25.7 & 0.233 \\
\hline $120 \mathrm{~min}$ & 6 & $38,41,44,59,60,70$ & $\begin{array}{l}-0.42(-0.82 \\
\text { to }-0.03)\end{array}$ & 0.034 & $\begin{array}{l}-0.42(-0.77 \\
\text { to }-0.06)\end{array}$ & 0.021 & 16.8 & 0.305 \\
\hline
\end{tabular}

Postprandial AG stratified by duration of postprandial period in normal-weight group after excluding the studies with heterogeneity

\begin{tabular}{|c|c|c|c|c|c|c|c|c|}
\hline $30 \mathrm{~min}$ & 3 & $70,73,78$ & $\begin{array}{l}-1.19(-1.54 \\
\text { to }-0.84)\end{array}$ & $<0.001$ & $\begin{array}{l}-1.19(-1.54 \\
\text { to }-0.84)\end{array}$ & $<0.001$ & 0.0 & 0.371 \\
\hline $60 \mathrm{~min}$ & 7 & $38,44,59,60,70,72,78$ & $\begin{array}{l}-0.75(-1.11 \\
\text { to }-0.39)\end{array}$ & $<0.001$ & $\begin{array}{l}-0.72(-1.02 \\
\text { to }-0.41)\end{array}$ & $<0.001$ & 25.7 & 0.233 \\
\hline $120 \mathrm{~min}$ & 6 & $38,41,44,59,60,70$ & $\begin{array}{l}-0.42(-0.82 \\
\text { to }-0.03)\end{array}$ & 0.034 & $\begin{array}{l}-0.42(-0.77 \\
\text { to }-0.06)\end{array}$ & 0.021 & 16.8 & 0.305 \\
\hline \multicolumn{9}{|c|}{ Postprandial AG stratified by duration of postprandial period in obese group } \\
\hline $30 \mathrm{~min}$ & 4 & $44,70,73,78$ & $\begin{array}{l}-0.61(-0.89 \\
\text { to }-0.34)\end{array}$ & $<0.001$ & $\begin{array}{l}-0.61(-0.89 \\
\text { to }-0.34)\end{array}$ & $<0.001$ & 0.0 & 0.762 \\
\hline $60 \mathrm{~min}$ & 7 & $38,44,59,60,70,72,78$ & $\begin{array}{l}-0.62(-0.94 \\
\text { to }-0.30)\end{array}$ & $<0.001$ & $\begin{array}{l}-0.62(-0.91 \\
\text { to }-0.33)\end{array}$ & $<0.001$ & 15.9 & 0.390 \\
\hline $120 \mathrm{~min}$ & 6 & $38,41,44,59,60,70$ & $-0.31(-0.68$ to 0.05$)$ & 0.092 & $-0.28(-0.60$ to 0.03$)$ & 0.074 & 24.0 & 0.254 \\
\hline
\end{tabular}

Table 5. Meta-analysis for comparison of postprandial AG levels stratified by duration of postprandial period (postprandial vs. baseline). $A G$ acyl ghrelin. 


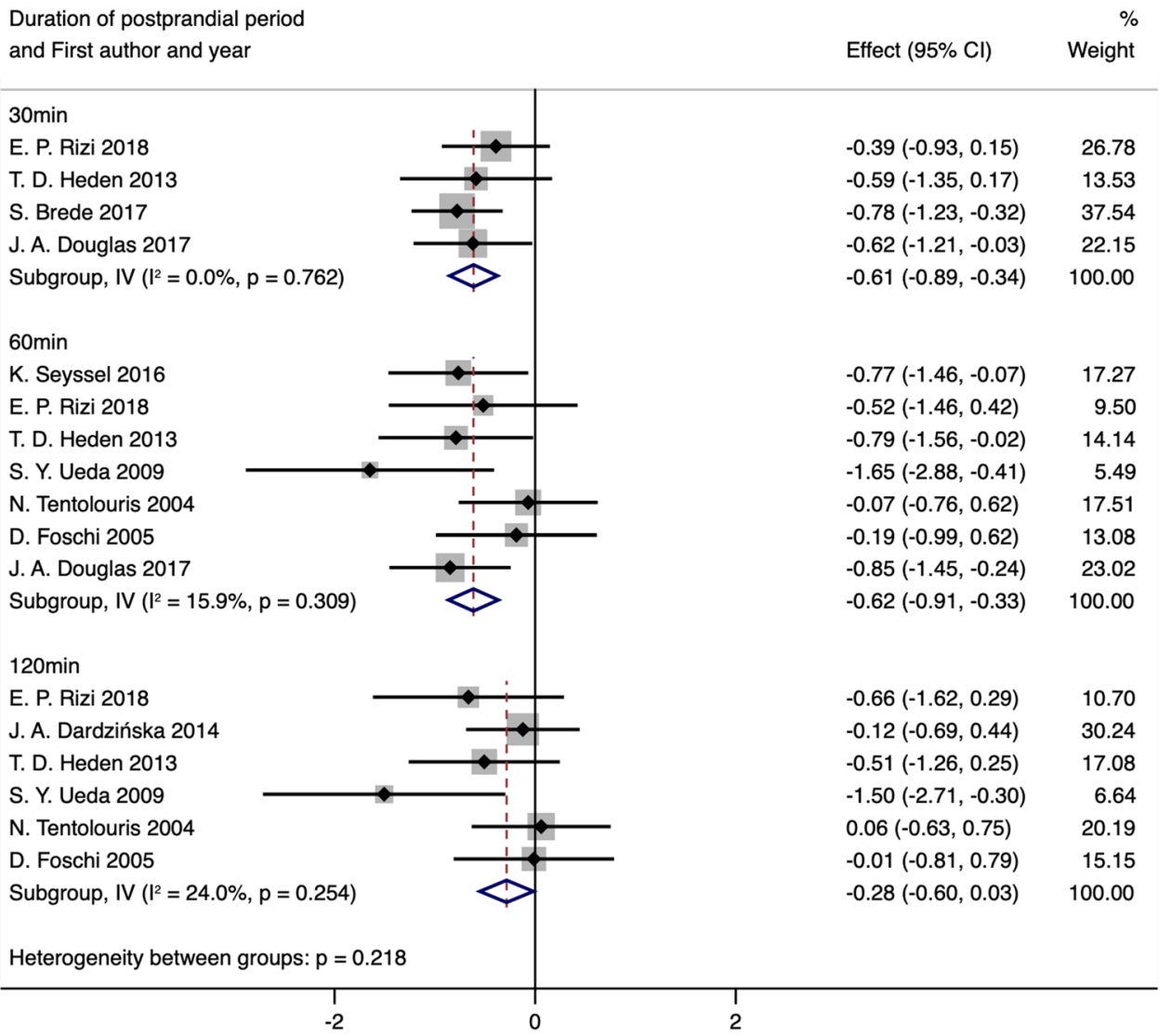

Figure 6. Forest plot for comparisons of postprandial AG levels stratified by duration of postprandial period in the obese group (postprandial vs. baseline).

Postprandial acyl ghrelin in obesity. When comparing the different concentrations of postprandial AG between obese subjects and controls, the former still maintained significantly lower levels at each time stratification (Fig. 4 and Table 4). These results also reveal that a high level of ghrelin is not an inherent feature of simple obesity. We observed a postprandial decline in AG, both in healthy and obese individuals (Figs. 5, 6 and Table 5), although several studies demonstrated a temporary elevation after the initiation of an eating episode ${ }^{72,92,93}$. This inconsistency can be attributed to the different time points we selected because the rapid postprandial fall in circulating ghrelin levels is most likely to be triggered after nutrient ingestion ${ }^{29}$, even though macronutrient composition is taken into consideration ${ }^{44}$ and a postprandial response of plasma ghrelin requires postgastric stimulation. A longer gastric transition time might cause a longer duration for ghrelin suppression.

When stratified by the included time points, the difference in AG concentrations between postprandial $120 \mathrm{~min}$ and baseline states in obesity disappeared (SMD obtained from fixed-effects model: $-0.28 ; 95 \%$ CI: -0.60 to $0.03 ; P_{\mathrm{SMD}}=0.074$, Table 5), suggesting a shorter duration of AG suppression in obese subjects after meal intake because the difference was still significant in healthy controls at this time point (SMD obtained from fixed-effects model: $-0.42 ; 95 \% \mathrm{CI}:-0.77$ to $-0.06 ; P_{\mathrm{SMD}}=0.021$, Table 5 ). However, independent estimation of the extent of AG decline reached a similar value between the obese and healthy groups, since the mean change between baseline and postprandial states was not significantly different between the two groups in each period (Fig. 7 and Table 6), which means that obese subjects possess a similar degree of postprandial ghrelin reduction as normal weight subjects (Fig. 8).

To date, the study of the ghrelin response to meal in the obese subjects showed controversial results. Even existing researches prefer a blunted postprandial ghrelin suppression ${ }^{33,59,94,95}$, our finding is consistent with studies which do show a similarly meal-induced suppression between obesity and normal ${ }^{44,96,97}$. This phenomenon illustrates the establishment of a new body weight set-point and an adaptation of energy homeostasis under obese states. The shorter duration of AG suppression may be attributed to the lowering of basal ghrelin levels, rapidly recovering the starvation level, shortening the food-free interval between meals and causing frequent eating. In view of this faster rebound in postprandial suppression, it is not hard to understand the reversal of obesity-induced ghrelin suppression under calorie restriction ${ }^{98}$, and anti-ghrelin therapy may be more suitable for those recovery stages than for those lower baseline periods. More work is needed to fully elucidate ghrelin's homeostasis, which will provide clues in therapeutic interventions for patients with metabolic diseases.

Limitations. When applying the results in this meta-analysis, several limitations should be carefully considered. First, a relatively limited number of subjects were included in the evaluation of different forms of ghrelin 
Duration of postprandial period and First author and year

Effect $(95 \% \mathrm{Cl}) \quad$ Weight

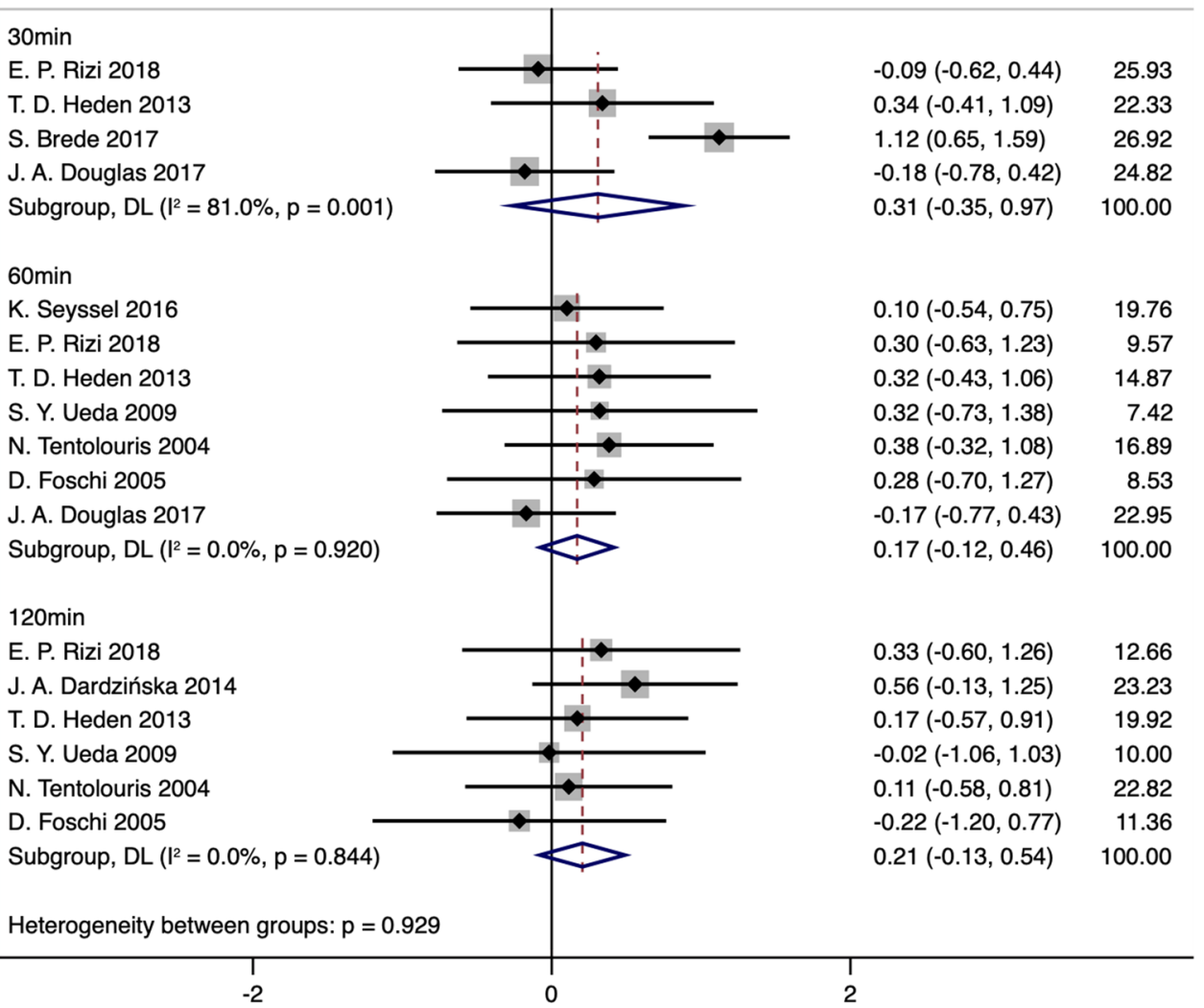

NOTE: Weights and between-subgroup heterogeneity test are from random-effects model

Figure 7. Forest plot of the changes in postprandial AG levels stratified by the duration of the postprandial period (obesity vs. normal weight).

\begin{tabular}{|c|c|c|c|c|c|c|c|c|}
\hline \multirow[b]{2}{*}{ Groups or subgroups } & \multirow[b]{2}{*}{$\mathbf{N}$} & \multirow[b]{2}{*}{ References } & \multicolumn{2}{|c|}{ Random-effects model } & \multicolumn{2}{|l|}{ Fix-effects model } & \multirow[b]{2}{*}{$I^{2}(\%)$} & \multirow[b]{2}{*}{$\boldsymbol{P}_{\text {Heterogeneit }}$} \\
\hline & & & SMD $(95 \% \mathrm{CI})$ & $\boldsymbol{P}_{\text {SMD }}$ & SMD (95\%CI) & $P_{\text {SMD }}$ & & \\
\hline \multicolumn{9}{|c|}{ Change of postprandial AG stratified by duration of postprandial period } \\
\hline $30 \mathrm{~min}$ & 4 & $44,70,73,78$ & $0.31(-0.35$ to 0.97$)$ & 0.359 & $0.38(0.10$ to 0.67$)$ & 0.008 & 81.0 & 0.001 \\
\hline $60 \mathrm{~min}$ & 7 & $38,44,59,60,70,72,78$ & $0.17(-0.12$ to 0.46$)$ & 0.246 & $0.17(-0.12$ to 0.46$)$ & 0.246 & 0.0 & 0.920 \\
\hline $120 \mathrm{~min}$ & 6 & $38,41,44,59,60,70$ & $0.21(-0.13$ to 0.54$)$ & 0.224 & $0.21(-0.13$ to 0.54$)$ & 0.224 & 0.0 & 0.884 \\
\hline \multicolumn{9}{|c|}{ Change of postprandial AG stratified by duration of postprandial period after excluding the studies with heterogeneity } \\
\hline $30 \mathrm{~min}$ & 3 & $44,70,78$ & $0.03(-0.38$ to 0.33$)$ & 0.887 & $0.03(-0.38$ to 0.33$)$ & 0.887 & 0.0 & 0.541 \\
\hline $60 \mathrm{~min}$ & 7 & $38,44,59,60,70,72,78$ & $0.17(-0.12$ to 0.46$)$ & 0.246 & $0.17(-0.12$ to 0.46$)$ & 0.246 & 0.0 & 0.920 \\
\hline $120 \mathrm{~min}$ & 6 & $38,41,44,59,60,70$ & $0.21(-0.13$ to 0.54$)$ & 0.224 & $0.21(-0.13$ to 0.54$)$ & 0.224 & 0.0 & 0.844 \\
\hline
\end{tabular}

Table 6. Meta-analysis of changes in postprandial AG levels stratified by duration of the postprandial period (obesity vs. normal weight). $A G$ acyl ghrelin.

independently between obese and lean healthy individuals, which might affect the statistical power. To expand the coverage of eligible studies, MetS patients were not excluded because abdominal obesity is one of the criteria to define metabolic syndrome ${ }^{50,51}$. However, metabolic comorbid conditions, including hypertension and IGT, could also affect ghrelin responses ${ }^{99,100}$. Second, the lack of sufficient data in these studies limited our further analysis, such as the postprandial DAG levels, AG/DAG ratio (a useful biomarker of excessive weight gain linked to obesity and diabetes), and AUC (area under the curve, an outcome representing overall hormone concentration over a specific time period in endocrinological studies). Furthermore, although Galbraith plots and subgroup analyses were used to explore heterogeneity, much of it remains to be explained and reported, including the varied types of mixed meals, different amounts of energy for meal tests, inconsistent duration of postprandial period, gender, ethnicity, age distribution and so on, and overestimating of pooled SMDs cannot be ignored. In 


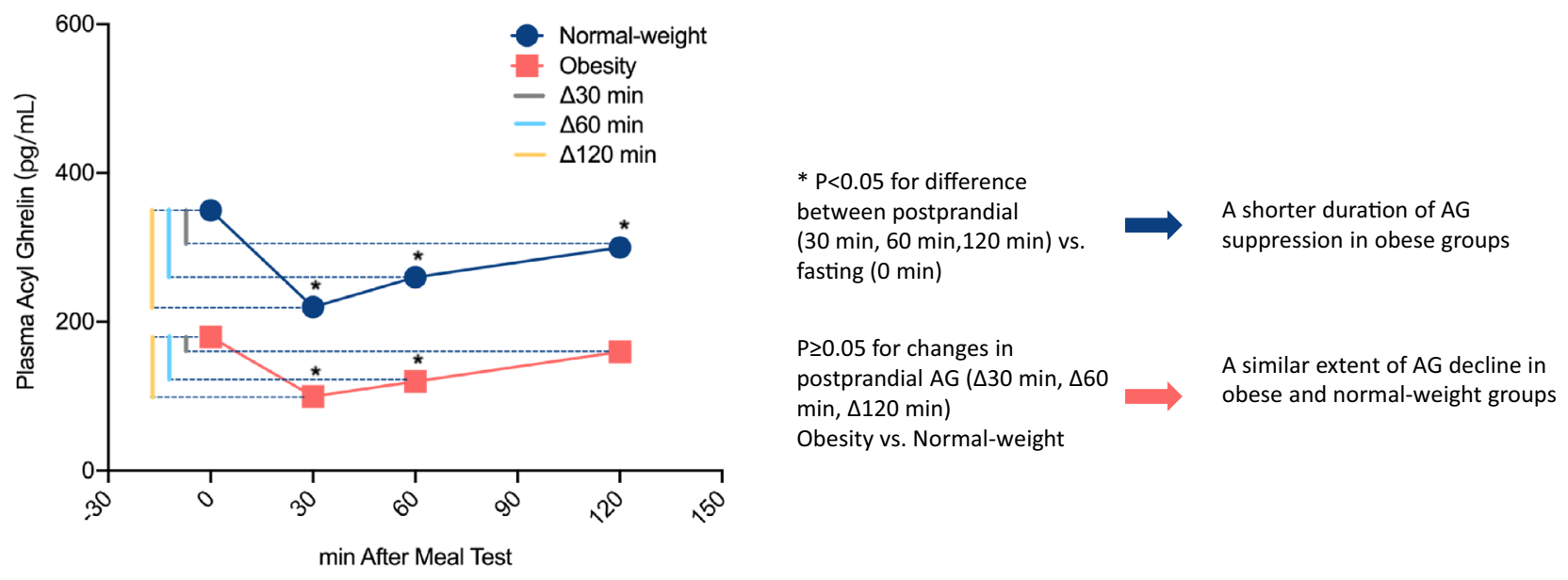

Figure 8. Diagram of the analysis of postprandial AG levels (the significance and AG levels reflect tendency only, cannot represent actual levels).

addition, the language of the included studies was constrained to English, which was partially responsible for the publication biases.

\section{Conclusion}

Taken together, our meta-analysis strengthens the clinical evidence supporting the following: lower baseline levels of circulating AG and DAG in obese individuals; the decline of postprandial circulating AG levels, both for healthy and obese individuals; and the shorter duration of AG suppression in obese subjects after meal intake. We support the existence of physiological adaptation in ghrelin under obesity, and the simultaneous decline in both AG and DAG is a symbol of positive energy balance. Despite some limitations in our study, we believe that this meta-analysis has significance for follow-up studies to elucidate the roles of various ghrelin forms in energy homeostasis. Furthermore, larger and more rigorous clinical trials with standardized test meals and fixed durations of the postprandial period are required to confirm these conclusions.

\section{Data availability}

No new data were created or analyzed in this study. Data sharing is not applicable to this article.

Received: 2 August 2021; Accepted: 1 February 2022

Published online: 17 February 2022

\section{References}

1. Centers for Disease Control and Prevention. Overweight \& Obesity. Defining Adult Overweight and Obesity. https://www.cdc.gov/ obesity/adult/defining.html.

2. Meldrum, D., Morris, M. \& Gambone, J. Obesity pandemic: Causes, consequences, and solutions-But do we have the will?. Fertil. Steril. 107, 833-839. https://doi.org/10.1016/j.fertnstert.2017.02.104 (2017).

3. Stefan, N., Birkenfeld, A. \& Schulze, M. Global pandemics interconnected-Obesity, impaired metabolic health and COVID-19. Nat. Rev. Endocrinol. 17, 135-149. https://doi.org/10.1038/s41574-020-00462-1 (2021).

4. Wren, A. et al. Ghrelin enhances appetite and increases food intake in humans. J. Clin. Endocrinol. Metab. 86, 5992. https://doi. org/10.1210/jcem.86.12.8111 (2001).

5. Cleverdon, E., McGovern-Gooch, K. \& Hougland, J. The octanoylated energy regulating hormone ghrelin: An expanded view of ghrelin's biological interactions and avenues for controlling ghrelin signaling. Mol. Membr. Biol. 33, 111-124. https://doi.org/ $10.1080 / 09687688.2017 .1388930(2016)$.

6. Müller, T. et al. Ghrelin. Mol. Metab. 4, 437-460. https://doi.org/10.1016/j.molmet.2015.03.005 (2015).

7. Tschöp, M., Smiley, D. \& Heiman, M. Ghrelin induces adiposity in rodents. Nature 407, 908-913. https://doi.org/10.1038/35038 $090(2000)$.

8. Akamizu, T. et al. Pharmacokinetics, safety, and endocrine and appetite effects of ghrelin administration in young healthy subjects. Eur. J. Endocrinol. 150, 447-455. https://doi.org/10.1530/eje.0.1500447 (2004).

9. Masuda, Y. et al. Ghrelin stimulates gastric acid secretion and motility in rats. Biochem. Biophys. Res. Commun. 276, 905-908. https://doi.org/10.1006/bbrc.2000.3568 (2000).

10. Date, Y. et al. Ghrelin, a novel growth hormone-releasing acylated peptide, is synthesized in a distinct endocrine cell type in the gastrointestinal tracts of rats and humans. Endocrinology 141, 4255-4261. https://doi.org/10.1210/endo.141.11.7757 (2000).

11. Kojima, M. et al. Ghrelin is a growth-hormone-releasing acylated peptide from stomach. Nature 402, 656-660. https://doi.org/ $10.1038 / 45230$ (1999).

12. Hosoda, H., Kojima, M., Matsuo, H. \& Kangawa, K. Ghrelin and des-acyl ghrelin: Two major forms of rat ghrelin peptide in gastrointestinal tissue. Biochem. Biophys. Res. Commun. 279, 909-913. https://doi.org/10.1006/bbrc.2000.4039 (2000).

13. Akamizu, T. et al. Separate measurement of plasma levels of acylated and desacyl ghrelin in healthy subjects using a new direct ELISA assay. J. Clin. Endocrinol. Metab. 90, 6-9. https://doi.org/10.1210/jc.2004-1640 (2005).

14. Gutierrez, J. et al. Ghrelin octanoylation mediated by an orphan lipid transferase. Proc. Natl. Acad. Sci. U. S. A. 105, 6320-6325. https://doi.org/10.1073/pnas.0800708105 (2008). 
15. Yang, J., Brown, M., Liang, G., Grishin, N. \& Goldstein, J. Identification of the acyltransferase that octanoylates ghrelin, an appetite-stimulating peptide hormone. Cell 132, 387-396. https://doi.org/10.1016/j.cell.2008.01.017 (2008).

16. Motoyasu, S., Yoshihiro, N., Junko, Y., Yoshiyuki, H. \& Hiroyuki, S. Identification and characterization of acyl-protein thioesterase 1/lysophospholipase I as a ghrelin deacylation/lysophospholipid hydrolyzing enzyme in fetal bovine serum and conditioned medium. Endocrinology 151, 4765-4775. https://doi.org/10.1210/en.2010-0412 (2010).

17. Chen, V. P., Gao, Y., Geng, L. \& Brimijoin, S. Butyrylcholinesterase gene transfer in obese mice prevents postdieting body weight rebound by suppressing ghrelin signaling. Proc. Natl. Acad. Sci. U. S. A. 114, 10960-10965. https://doi.org/10.1073/pnas. 17065 17114 (2017).

18. Chen, V. P., Gao, Y., Geng, L. \& Brimijoin, S. Butyrylcholinesterase regulates central ghrelin signaling and has an impact on food intake and glucose homeostasis. Int. J. Obes. 41, 1413-1419. https://doi.org/10.1038/ijo.2017.123 (2017).

19. Fernandez, G., Cabral, A., Cornejo, M. P., Francesco, P. \& Perello, M. Des-acyl ghrelin directly targets the arcuate nucleus in a ghrelin-receptor independent manner and impairs the orexigenic effect of ghrelin. J. Neuroendocrinol. 28, 1-12. https://doi.org/ $10.1111 /$ jne.12349 (2015).

20. Koji, T. et al. Des-acyl ghrelin induces food intake by a mechanism independent of the growth hormone secretagogue receptor. Endocrinology 147, 2306-2314. https://doi.org/10.1210/en.2005-1357 (2006).

21. Delhanty, P. J., Neggers, S. J. \& Lely, A. Des-acyl ghrelin: A metabolically active peptide. Endocr. Dev. 25, 112-121. https://doi. org/10.1159/000346059 (2013)

22. Broglio, F. et al. Non-acylated ghrelin counteracts the metabolic but not the neuroendocrine response to acylated ghrelin in humans. J. Clin. Endocrinol. Metab. 89, 3062-3065. https://doi.org/10.1210/jc.2003-031964 (2004).

23. Heppner, K. et al. Both acyl and des-acyl ghrelin regulate adiposity and glucose metabolism via central nervous system ghrelin receptors. Diabetes 63, 122-131. https://doi.org/10.2337/db13-0414 (2014).

24. Hiroyuki, A. et al. Transgenic mice overexpressing des-acyl ghrelin show small phenotype. Endocrinology 146, 355-364. https:// doi.org/10.1210/en.2004-0629 (2005).

25. Delhanty, P. J. D. et al. Unacylated ghrelin rapidly modulates lipogenic and insulin signaling pathway gene expression in metabolically active tissues of GHSR deleted mice. PLOS ONE 5, e11749. https://doi.org/10.1371/journal.pone.0011749 (2010).

26. Cui, H., López, M. \& Rahmouni, K. The cellular and molecular bases of leptin and ghrelin resistance in obesity. Nat. Rev. Endocrinol. 13, 338-351. https://doi.org/10.1038/nrendo.2016.222 (2017).

27. Yin, X., Li, Y., Xu, G., An, W. \& Zhang, W. Ghrelin fluctuation, what determines its production?. Acta Biochim. Biophys. Sin. 41, 188-197. https://doi.org/10.1093/abbs/gmp001 (2009).

28. Ariyasu, H. et al. Stomach is a major source of circulating ghrelin, and feeding state determines plasma ghrelin-like immunoreactivity levels in humans. J. Clin. Endocrinol. Metab. 86, 4753-4758. https://doi.org/10.1210/jcem.86.10.7885 (2001).

29. Cummings, D. et al. A preprandial rise in plasma ghrelin levels suggests a role in meal initiation in humans. Diabetes 50, 1714-1719. https://doi.org/10.2337/diabetes.50.8.1714 (2001).

30. Mani, B. \& Zigman, J. Ghrelin as a survival hormone. Trends Endocrinol. Metab. 28, 843-854. https://doi.org/10.1016/j.tem. 2017.10.001 (2017).

31. Tschop \& Matthias. Circulating ghrelin levels are decreased in human obesity. Diabetes 50, 707-709. https://doi.org/10.2337/ diabetes.50.4.707 (2001).

32. Shiiya, T. et al. Plasma ghrelin levels in lean and obese humans and the effect of glucose on ghrelin secretion. J. Clin. Endocrinol. Metab. 87, 240-244. https://doi.org/10.1210/jcem.87.1.8129 (2002).

33. le Roux, C. et al. Postprandial plasma ghrelin is suppressed proportional to meal calorie content in normal-weight but not obese subjects. J. Clin. Endocrinol. Metab. 90, 1068-1071. https://doi.org/10.1210/jc.2004-1216 (2005).

34. Delhanty, P. et al. The acylated (AG) to unacylated (UAG) ghrelin ratio in esterase inhibitor-treated blood is higher than previously described. Clin. Endocrinol. 82, 142-146. https://doi.org/10.1111/cen.12489 (2015).

35. Deschaine, S. \& Leggio, L. Understanding plasma treatment effect on human acyl-ghrelin concentrations. Eur. Rev. Med. Pharmacol. Sci. 24, 1585-1589. https://doi.org/10.26355/eurrev_202002_20216 (2020).

36. Delhanty, P., Neggers, S. \& van der Lely, A. Should we consider des-acyl ghrelin as a separate hormone and if so, what does it do?. Front. Horm. Res. 42, 163-174. https://doi.org/10.1159/000358345 (2014).

37. Marzullo, P. et al. Acylated ghrelin decreases during acute exercise in the lean and obese state. Clin. Endocrinol. (Oxf.) 69, 970-971. https://doi.org/10.1111/j.1365-2265.2008.03275.x (2008).

38. Ueda, S. Y. et al. Changes in gut hormone levels and negative energy balance during aerobic exercise in obese young males. J. Endocrinol. 201, 151-159. https://doi.org/10.1677/joe-08-0500 (2009).

39. Homaee, H. M., Moradi, F., Azarbayjani, M. A. \& Peeri, M. Relationships between acylated ghrelin with growth hormone, insulin resistance, lipid profile, and cardio respiratory function in lean and obese men. J. Res. Med. Sci. 16, 1612-1618 (2011).

40. Rodríguez, A. et al. The ghrelin O-acyltransferase-ghrelin system reduces TNF- $\alpha$-induced apoptosis and autophagy in human visceral adipocytes. Diabetologia 55, 3038-3050. https://doi.org/10.1007/s00125-012-2671-5 (2012).

41. Dardzińska, J. et al. Fasting and postprandial acyl and desacyl ghrelin levels in obese and non-obese subjects. Endokrynol. Pol. 65, 377-381. https://doi.org/10.5603/ep.2014.0052 (2014).

42. Tamboli, R. A. et al. Metabolic responses to exogenous ghrelin in obesity and early after Roux-en-Y gastric bypass in humans. Diabetes Obes. Metab. 19, 1267-1275. https://doi.org/10.1111/dom.12952 (2017).

43. Kołodziejski, P. A. et al. Serum levels of spexin and kisspeptin negatively correlate with obesity and insulin resistance in women. Physiol. Res. 67, 45-56. https://doi.org/10.33549/physiolres.933467 (2018).

44. Rizi, E. P. et al. A high carbohydrate, but not fat or protein meal attenuates postprandial ghrelin, PYY and GLP-1 responses in Chinese men. PLoS ONE 13, 1-12. https://doi.org/10.1371/journal.pone.0191609 (2018).

45. Nakazato, M. et al. A role for ghrelin in the central regulation of feeding. Nature 409, 194-198. https://doi.org/10.1038/35051 587 (2001).

46. Altabas, V. \& Zjačić-Rotkvić, V. Anti-ghrelin antibodies in appetite suppression: Recent advances in obesity pharmacotherapy. ImmunoTargets Ther. 4, 123-130. https://doi.org/10.2147/itt.S60398 (2015).

47. Colon-Gonzalez, F., Kim, G., Lin, J., Valentino, M. \& Waldman, S. Obesity pharmacotherapy: What is next?. Mol. Aspects Med. 34, 71-83. https://doi.org/10.1016/j.mam.2012.10.005 (2013).

48. Hainer, V. Overview of new antiobesity drugs. Expert Opin. Pharmacother. 15, 1975-1978. https://doi.org/10.1517/14656566. 2014.946904 (2014).

49. Liang, Y., Yin, W., Yin, Y. \& Zhang, W. Ghrelin based therapy of metabolic diseases. Curr. Med. Chem. 28, 2565-2576. https:// doi.org/10.2174/0929867327666200615152804 (2021).

50. Expert Panel on Detection, Evaluation, and Treatment of High Blood Cholesterol in Adults. Executive Summary of the Third Report of The National Cholesterol Education Program (NCEP) Expert Panel on Detection, Evaluation, and Treatment of High Blood Cholesterol in Adults (Adult Treatment Panel III). JAMA 285, 2486-2497. https://doi.org/10.1001/jama.285.19.2486 (2001).

51. National Cholesterol Education Program (NCEP) Expert Panel on Detection, Evaluation, and Treatment of High Blood Cholesterol in Adults (Adult Treatment Panel III). Third Report of the National Cholesterol Education Program (NCEP) Expert Panel on Detection, Evaluation, and Treatment of High Blood Cholesterol in Adults (Adult Treatment Panel III) Final Report. Circulation 106, 3143-3421 (2002). 
52. Stang, A. Critical evaluation of the Newcastle-Ottawa scale for the assessment of the quality of nonrandomized studies in metaanalyses. Eur. J. Epidemiol. 25, 603-605. https://doi.org/10.1007/s10654-010-9491-z (2010).

53. McGrath, S., Zhao, X., Steele, R., Thombs, B. D. \& Benedetti, A. Estimating the sample mean and standard deviation from commonly reported quantiles in meta-analysis. Stat. Methods Med. Res. 29, 2520-2537. https://doi.org/10.1177/0962280219889080 (2020).

54. Barazzoni, R. et al. Plasma total and unacylated ghrelin predict 5-year changes in insulin resistance. Clin. Nutr. 35, 1168-1173. https://doi.org/10.1016/j.clnu.2015.10.002 (2016).

55. Liu, X., Guo, Y., Li, Z. \& Gong, Y. The role of acylated ghrelin and unacylated ghrelin in the blood and hypothalamus and their interaction with nonalcoholic fatty liver disease. Iran. J. Basic Med. Sci. 23, 1191-1196. https://doi.org/10.22038/ijbms.2020. 45356.10555 (2020).

56. Borenstein, M., Hedges, L. V., Higgins, J. \& Rothstein, H. R. Chapter 4: Effect sizes based on means. In Introduction to MetaAnalysis 21-32 https://doi.org/10.1002/9780470743386.ch4(Wiley, 2009).

57. Chapter 6: Choosing Effect Measures and Computing Estimates of Effect. Cochrane Handbook for Systematic Reviews of Interventions. https://training.cochrane.org/handbook/current/chapter-06 (Version 6.1, 2020).

58. Marzullo, P. et al. The relationship between active ghrelin levels and human obesity involves alterations in resting energy expenditure. J. Clin. Endocrinol. Metab. 89, 936-939. https://doi.org/10.1210/jc.2003-031328 (2004).

59. Tentolouris, N. et al. Differential effects of high-fat and high-carbohydrate content isoenergetic meals on plasma active ghrelin concentrations in lean and obese women. Horm. Metab. Res. 36, 559-563. https://doi.org/10.1055/s-2004-825761 (2004).

60. Foschi, D. et al. Vertical banded gastroplasty modifies plasma ghrelin secretion in obese patients. Obes. Surg. 15, 1129-1132. https://doi.org/10.1381/0960892055002338 (2005).

61. Suematsu, M. et al. Decreased circulating levels of active ghrelin are associated with increased oxidative stress in obese subjects. Eur. J. Endocrinol. 153, 403-407. https://doi.org/10.1530/eje.1.01977 (2005).

62. Baranowska, B. et al. Neuroendocrine control of metabolic homeostasis in Polish centenarians. J. Physiol. Pharmacol. 57, 55-61. https://doi.org/10.1152/jn.00538.2006 (2006).

63. Bik, W. et al. The relationship between metabolic status and levels of adiponectin and ghrelin in lean women with polycystic ovary syndrome. Gynecol. Endocrinol. 23, 325-331. https://doi.org/10.1080/09513590701260169 (2007).

64. Zwirska-Korczala, K. et al. Basal and postprandial plasma levels of PYY, ghrelin, cholecystokinin, gastrin and insulin in women with moderate and morbid obesity and metabolic syndrome. J. Physiol. Pharmacol. 58, 13-35 (2007).

65. Nakahara, T. et al. Plasma obestatin concentrations are negatively correlated with body mass index, insulin resistance index, and plasma leptin concentrations in obesity and anorexia nervosa. Biol. Psychiatry 64, 252-255. https://doi.org/10.1016/j.biops ych.2007.08.005 (2008).

66. Carroll, J. F., Franks, S. F., Smith, A. B. \& Phelps, D. R. Visceral adipose tissue loss and insulin resistance 6 months after laparoscopic gastric banding surgery: A preliminary study. Obes. Surg. 19, 47-55. https://doi.org/10.1007/s11695-008-9642-4 (2009).

67. Dunn, J. P. et al. Relationship of dopamine type 2 receptor binding potential with fasting neuroendocrine hormones and insulin sensitivity in human obesity. Diabetes Care 35, 1105-1111. https://doi.org/10.2337/dc11-2250 (2012).

68. Arafat, A. M. et al. The impact of insulin-independent, glucagon-induced suppression of total ghrelin on satiety in obesity and type 1 diabetes mellitus. J. Clin. Endocrinol. Metab. 98, 4133-4142. https://doi.org/10.1210/jc.2013-1635 (2013).

69. Haluzíková, D. et al. Laparoscopic sleeve gastrectomy differentially affects serum concentrations of FGF-19 and FGF-21 in morbidly obese subjects. Obesity (Silver Spring) 21, 1335-1342. https://doi.org/10.1002/oby.20208 (2013).

70. Heden, T. D., Liu, Y., Park, Y., Dellsperger, K. C. \& Kanaley, J. A. Acute aerobic exercise differentially alters acylated ghrelin and perceived fullness in normal-weight and obese individuals. J. Appl. Physiol. 1985(115), 680-687. https://doi.org/10.1152/jappl physiol.00515.2013 (2013)

71. Savage, S. W. et al. Regulation of novelty seeking by midbrain dopamine D2/D3 signaling and ghrelin is altered in obesity. Obesity 22, 1452-1457. https://doi.org/10.1002/oby.20690 (2014).

72. Seyssel, K. et al. Plasma acyl-ghrelin increases after meal initiation: A new insight. Eur. J. Clin. Nutr. 70, 790-794. https://doi. org/10.1038/ejcn.2015.181 (2016).

73. Brede, S. et al. Visual food cues decrease postprandial glucose concentrations in lean and obese men without affecting food intake and related endocrine parameters. Appetite 117, 255-262. https://doi.org/10.1016/j.appet.2017.07.001 (2017).

74. Ezquerro, S. et al. Ghrelin reduces TNF-alpha-induced human hepatocyte apoptosis, autophagy, and pyroptosis: Role in obesityassociated NAFLD. J. Clin. Endocrinol. Metab. 104, 21-37. https://doi.org/10.1210/jc.2018-01171 (2019).

75. Iceta, S. et al. Ghrelin concentration as an indicator of eating-disorder risk in obese women. Diabetes Metab. 45, 160-166. https:// doi.org/10.1016/j.diabet.2018.01.006 (2019).

76. Yunker, A. G. et al. Appetite-regulating hormones are reduced after oral sucrose vs glucose: Influence of obesity, insulin resistance, and sex. J. Clin. Endocrinol. Metab. 106, 654-664. https://doi.org/10.1210/clinem/dgaa865 (2021).

77. Lopez-Aguilar, I., del Rocio Ibarra-Reynoso, L. \& Manuel Malacara, J. Association of nesfatin-1, acylated ghrelin and cortisol with scores of compulsion, food addiction, and binge eating in adults with normal weight and with obesity. Ann. Nutr. Metab. 73, 54-61. https://doi.org/10.1159/000490357 (2018).

78. Douglas, J. A. et al. Acute effects of exercise on appetite, ad libitum energy intake and appetite-regulatory hormones in lean and overweight/obese men and women. Int. J. Obes. (Lond.) 41, 1737-1744. https://doi.org/10.1038/ijo.2017.181 (2017).

79. Ozkan, Y. et al. Acylated and desacylated ghrelin, preptin, leptin, and nesfatin-1 peptide changes related to the body mass index. Int. J. Endocrinol. 2013, 236085. https://doi.org/10.1155/2013/236085 (2013).

80. Nogueira, J. P. et al. Unacylated ghrelin is associated with the isolated low HDL-cholesterol obese phenotype independently of insulin resistance and CRP level. Nutr. Metab. (Lond.) 9, 17. https://doi.org/10.1186/1743-7075-9-17 (2012).

81. Gelisgen, R. et al. Effects of laparoscopic gastric band applications on plasma and fundic acylated ghrelin levels in morbidly obese patients. Obes. Surg. 22, 299-305. https://doi.org/10.1007/s11695-011-0498-7 (2012).

82. Karcz-Socha, I., Zwirska-Korczala, K., Zembala, M., Borgiel-Marek, H. \& Karcz, W. K. Ghrelin PYY 3-36 serum changes in left ventricular hypertrophic, insulin-resistant, hypertensive obese patients. Obes. Facts 4, 386-392. https://doi.org/10.1159/00033 4198 (2011).

83. Krzyzanowska-Swiniarska, B., Kempa, A., Miazgowski, T. \& Pilarska, K. Serum acylated ghrelin, adiponectin and leptin levels in normal-weight and obese premenopausal women. Horm. Metab. Res. 39, 835-839. https://doi.org/10.1055/s-2007-991175 (2007).

84. Gauna, C. et al. Administration of acylated ghrelin reduces insulin sensitivity, whereas the combination of acylated plus unacylated ghrelin strongly improves insulin sensitivity. J. Clin. Endocrinol. Metab. 89, 5035-5042. https://doi.org/10.1210/jc. 2004-0363 (2004).

85. Abizaid, A. \& Hougland, J. Ghrelin signaling: GOAT and GHS-R1a take a LEAP in complexity. Trends Endocrinol. Metab. 31, 107-117. https://doi.org/10.1016/j.tem.2019.09.006 (2020).

86. Monteleone, P. et al. Circulating ghrelin is decreased in non-obese and obese women with binge eating disorder as well as in obese non-binge eating women, but not in patients with bulimia nervosa. Psychoneuroendocrinology 30, 243-250. https://doi. org/10.1016/j.psyneuen.2004.07.004 (2005).

87. Hernandez, D., Mehta, N. \& Geliebter, A. Meal-related acyl and des-acyl ghrelin and other appetite-related hormones in people with obesity and binge eating. Obesity (Silver Spring) 27, 629-635. https://doi.org/10.1002/oby.22431 (2019). 
88. Nunez-Salces, M., Li, H., Feinle-Bisset, C., Young, R. \& Page, A. The regulation of gastric ghrelin secretion. Acta Physiol. (Oxf. Engl.) 231, e13588. https://doi.org/10.1111/apha.13588 (2021).

89. Yanagi, S., Sato, T., Kangawa, K. \& Nakazato, M. The homeostatic force of ghrelin. Cell Metab. 27, 786-804. https://doi.org/10. 1016/j.cmet.2018.02.008 (2018).

90. Sakata, I. et al. Glucose-mediated control of ghrelin release from primary cultures of gastric mucosal cells. Am. J. Physiol. Endocrinol. Metab. 302, E1300-E1310. https://doi.org/10.1152/ajpendo.00041.2012 (2012).

91. Zigman, J., Bouret, S. \& Andrews, Z. Obesity impairs the action of the neuroendocrine ghrelin system. Trends Endocrinol. Metab. 27, 54-63. https://doi.org/10.1016/j.tem.2015.09.010 (2016).

92. Erdmann, J., Hebeisen, Y., Lippl, F., Wagenpfeil, S. \& Schusdziarra, V. Food intake and plasma ghrelin response during potato-, rice- and pasta-rich test meals. Eur. J. Nutr. 46, 196-203. https://doi.org/10.1007/s00394-007-0649-8 (2007).

93. Karine, S., Esra, T., Rachel, L., Neal, S. \& Eve, V. C. Twenty-four-hour profiles of acylated and total ghrelin: Relationship with glucose levels and impact of time of day and sleep. J. Clin. Endocrinol. Metab. 96, 486-493. https://doi.org/10.1210/jc.2010-1978 (2011).

94. English, P. J., Ghatei, M. A., Malik, I. A., Bloom, S. R. \& Wilding, J. Food fails to suppress ghrelin levels in obese humans. J. Clin. Endocrinol. Metab. 87, 2984-2987. https://doi.org/10.1210/jcem.87.6.8738 (2002).

95. Koliaki, C., Liatis, S., Dalamaga, M. \& Kokkinos, A. The implication of gut hormones in the regulation of energy homeostasis and their role in the pathophysiology of obesity. Curr. Obes. Rep. 9, 255-271. https://doi.org/10.1007/s13679-020-00396-9 (2020).

96. Brennan, I. et al. Effects of fat, protein, and carbohydrate and protein load on appetite, plasma cholecystokinin, peptide YY, and ghrelin, and energy intake in lean and obese men. Am. J. Physiol. Gastrointest. Liver Physiol. 303, G129-G140. https://doi.org/ 10.1152/ajpgi.00478.2011 (2012).

97. Cummings, D. et al. Plasma ghrelin levels after diet-induced weight loss or gastric bypass surgery. N. Engl. J. Med. 346, 1623-1630. https://doi.org/10.1056/NEJMoa012908 (2002).

98. Briggs, D. et al. Calorie-restricted weight loss reverses high-fat diet-induced ghrelin resistance, which contributes to rebound weight gain in a ghrelin-dependent manner. Endocrinology 154, 709-717. https://doi.org/10.1210/en.2012-1421 (2013).

99. Huang, L. et al. Increased acyl ghrelin but decreased total ghrelin and unacyl ghrelin in Chinese Han people with impaired fasting glucose combined with impaired glucose tolerance. Peptides 60, 86-94. https://doi.org/10.1016/j.peptides.2014.07.022 (2014).

100. Wang, W. M. et al. Ghrelin and obestatin levels in hypertensive obese patients. J. Int. Med. Res. 42, 1202-1208. https://doi.org/ $10.1177 / 0300060514543040$ (2014).

\title{
Author contributions
}

Y.W., Y.C. and X.L. conceived and designed the study. Y.W., Q.W. and Q.C. conducted the literature searches and collected the data. Q.Z. performed the statistical analyses. Y.W. and Q.W. wrote the manuscript. Y.C. and Q.C. contributed to the article revision. All authors read and approved the final manuscript.

\section{Funding}

This present research was supported by the Subsidy Funds for Improving Medical Services and Guarantee Capabilities (Major Incurable Diseases) (CYW2019079) and Youth Science and Technology Innovation Project of Science and Technology Department of Sichuan Province (Grant No. 2021049). The funders had no role in the study design, data collection and analysis, decision to publish, or preparation of the manuscript.

\section{Competing interests}

The authors declare no competing interests.

Additional information

Supplementary Information The online version contains supplementary material available at https://doi.org/ 10.1038/s41598-022-06636-3.

Correspondence and requests for materials should be addressed to Q.C.

Reprints and permissions information is available at www.nature.com/reprints.

Publisher's note Springer Nature remains neutral with regard to jurisdictional claims in published maps and institutional affiliations.

\begin{abstract}
Open Access This article is licensed under a Creative Commons Attribution 4.0 International License, which permits use, sharing, adaptation, distribution and reproduction in any medium or format, as long as you give appropriate credit to the original author(s) and the source, provide a link to the Creative Commons licence, and indicate if changes were made. The images or other third party material in this article are included in the article's Creative Commons licence, unless indicated otherwise in a credit line to the material. If material is not included in the article's Creative Commons licence and your intended use is not permitted by statutory regulation or exceeds the permitted use, you will need to obtain permission directly from the copyright holder. To view a copy of this licence, visit http://creativecommons.org/licenses/by/4.0/.
\end{abstract}

(C) The Author(s) 2022 